\title{
AF9 promotes hESC neural differentiation through recruiting TET2 to neurodevelopmental gene loci for methylcytosine hydroxylation
}

\author{
Yunbo Qiao ${ }^{1, *}$, Xiongjun Wang ${ }^{2, *}$, Ran Wang ${ }^{1}$, Yuanyuan $\mathrm{Li}^{1}$, Fang $\mathrm{Yu}^{1}$, Xianfa Yang ${ }^{1,3}$, Lu Song ${ }^{1}$, \\ Guoliang $\mathrm{Xu}^{4}$, Y Eugene Chin ${ }^{2}$, Naihe Jing ${ }^{1}$ \\ ${ }^{1}$ State Key Laboratory of Cell Biology, Institute of Biochemistry and Cell Biology, Shanghai Institutes for Biological Sciences, \\ Chinese Academy of Sciences, Shanghai, China; ${ }^{2}$ Key Laboratory of Stem Cell Biology, Institute of Health Sciences, Shanghai \\ Institutes for Biological Sciences, Chinese Academy of Sciences/Shanghai Jiao Tong University School of Medicine, Shanghai, \\ China; ${ }^{3}$ School of Life Science and Technology, Shanghai Tech University, Shanghai, China; ${ }^{4}$ State Key Laboratory of \\ Molecular Biology, Institute of Biochemistry and Cell Biology, Shanghai Institutes for Biological Sciences, Chinese Academy of \\ Sciences, Shanghai, China
}

AF9 mutations have been implicated in human neurodevelopmental diseases and murine Af9 mediates histone methylation during cortical neuron generation. However, AF9 function and related mechanisms in human neurodevelopment remain unknown. Here we show that AF9 is necessary and sufficient for human embryonic stem cell (hESC) neural differentiation and neurodevelopmental gene activation. The 5-methylcytosine $(5 \mathrm{mC})$ dioxygenase TET2, which was identified in an AF9-associated protein complex, physically interacted with AF9. Both AF9 and TET2 co-localized in 5-hydroxymethylcytosine $(5 \mathrm{hmC})$-positive $\mathrm{hESC}$-derived neurons and were required for appropriate hESC neural differentiation. Upon binding to AAC-containing motifs, AF9 recruited TET2 to occupy the common neurodevelopmental gene loci to direct $5 \mathrm{mC}$-to-5hmC conversion, which was followed by sequential activation of neural target genes and hESC neural commitment. These findings define an AF9-TET2 regulatory complex for modulating human neural development and reveal a novel mechanism by which the AF9 recognition specificity and TET2 hydroxylation activity cooperate to control neurodevelopmental gene activation.

Keywords: AF9; TET2; human embryonic stem cell; neural differentiation; 5-hydroxymethylcytosine Cell Discovery (2015) 1, 15017; doi:10.1038/celldisc.2015.17; published online 28 July 2015

\section{Introduction}

The human central nervous system (CNS) is immensely complex with composition of precisely interconnected neurons. Previous studies have mainly focused on neuronal migration, subtype differentiation, and circuit formation in human neurocortex development [1-3]. The in vitro neural differentiation of human

*These authors contributed equally to this work.

Correspondence: Naihe Jing

Tel: +86 21 54921381; Fax: +86 21 54921011;

E-mail: njing@sibcb.ac.cn

or Y Eugene Chin

Tel: +86 21 63843432; Fax: 8621 63852655;

E-mail: yechin@sibs.ac.cn

Received 15 May 2015; accepted 25 May 2015 embryonic stem cells (hESCs) recapitulates human neural development with the presence of neural tubelike structures [4, 5]. Crucial factors, such as LIN-28 and $\mathrm{FOXO} 4$, have been revealed to participate in the regulation of hESC neural commitment $[6,7]$. In the mouse and other animal models, multiple neurodevelopmental genes such as ZNF521, SOX5, PAX6, $M A S H 1, N G N 2$, and $B R N 2$, have been uncovered [8-12]. The transcriptional activity of these key factors largely relies on the epigenetic regulation, which has important roles in neural fate specification to guarantee the normal human neurodevelopment [13]. However, the epigenetic mechanisms underlying human neural gene activation and lineage commitment remain elusive.

AF9 (also known as MLLT3), a histone methyltransferase and MLL fusion partner, is expressed in 
various CNS structures and is required for mouse embryonic patterning and cerebral cortex development [14-16]. Notably, human AF9 mutations are associated with neurodevelopmental diseases, such as mental retardation, epilepsy, and ataxia $[17,18]$. Nevertheless, the function of AF9 in human neural development remains unclear. Furthermore, AF9 mediates transcriptional activation through interactions with distinct factors in different cellular processes [19-21]. AF9 also acts as an epigenetic modifier to modulate histone methylation at target gene promoters [14, 22, 23]. The report that the MLL-AF9 fusion protects $H O X A 9$ from DNA methylation in leukemia [24] suggests that AF9 may participate in the regulation of DNA modification. The numerous studies describing the implications of DNA methylation and hydroxylation in neural development and neurological disorders [25-27] promote us to investigate the mechanistic role of AF9-mediated DNA modification in human neural development.

TET2 is a 5 -methycytosine $(5 \mathrm{mC})$ dioxygenase that catalyzes the conversion of $5 \mathrm{mC}$ to 5-hydroxymethylcytosine $(5 \mathrm{hmC})$ [28]. TET-mediated $5 \mathrm{mC}$ oxidation and DNA demethylation, which regulate gene expression and maintain cellular identity [29], are tightly correlated with neurodevelopment across species. TET1, another MLL fusion partner, promotes active DNA demethylation through hydroxylation in the mouse adult brain $[30,31]$. In the mouse CNS, $5 \mathrm{hmC}$ is present in Purkinje neurons and the brain [32] and co-localizes with NeuN in the cerebellum to mediate epigenetic dynamics during postnatal neurodevelopment [33]. The conserved features of $5 \mathrm{hmC}$ in mouse brain are displayed in a $5 \mathrm{mC}$ demethylation activity-dependent and region-specific manner relying on TET2 activity, and $5 \mathrm{hmC}$ is also associated with neurodevelopmental genes in the developing human cerebellum [33-36]. Moreover, the disruption of DNA methylation-associated processes results in diverse neurological disorders [32]. In addition, $5 \mathrm{hmC}$ enrichment and marked TET2 upregulation during neurogenesis were observed in the fetal cortex of the human brain [37]. It has also been reported that Tet3 directly regulates key neural gene expression in the Xenopus eye and neural development in a dioxygenase activity-dependent manner [38]. Nonetheless, the roles of TET 2 and $5 \mathrm{hmC}$ in human neurodevelopment are not fully understood. Importantly, how TET-mediated epigenetic regulation specifies neural gene activation and lineage commitment in human neural development remains largely unknown.

Here we show that both AF9 and TET2 are required for hESC neural differentiation. Mechanistically, AF9 physically interacts with TET2, and as a complex they bind to common neural-target gene loci to promote $5 \mathrm{mC}$-to-5hmC conversion and neurodevelopmental gene activation. We further demonstrate that the TET2 occupancy at neural gene loci is guided by AF9 that recognizes AAC-containing motifs. This study provides evidence that the target specificity of TET2 is determined by the epigenetic modifier AF9 during hESC neural differentiation, thereby linking the function of AF9-TET2 complex to human neural development.

\section{Results}

\section{AF9 is required for hESC neural differentiation}

To understand the biological function of AF9 in human neural development, hESCs were induced to differentiate into neural cells as previously described (Figure 1a) [5]. During in vitro hESC neural differentiation, these cells underwent a temporal course with morphological features, such as the neural tube-like rosettes. To identify the neural mediators involved in this process, the global expression profile of differentiating hESCs was measured every other day using RNA-seq analysis and multiple epigenetic factors with specific expression patterns were identified (unpublished data). During the progression of neural commitment, pluripotency genes (OCT4, TDGF1, NANOG, FOXD3, and TERT) gradually decreased and key neural regulators (SOX1, PAX6, and ZNF521) and $A F 9$ exhibited transcriptional upregulation (Figure 1a). Consistent with previous report [5], neurons derived using this protocol expressed forebrain markers (FOXG1, EMX1, and OTX2) (Figure 1a). Quantitative RT-PCR (qPCR) was performed to verify the observations in RNA-seq assays. AF9 upregulation coincided with the activation of neural progenitor (NP) genes (SOX1/5, ZNF521, and PAX6), proneural genes (MASH1, NGN2, NEUROG1, and NEUROD1/2/4), and neuronal markers (TUJ1 and MAP2) (Figure 1b; Supplementary Figure S1A). Notably, activation of these neural regulators followed a sequential neural fate determination process, and AF9 expression gradually increased from day 6 (Figure 1b), at the early stage of neural commitment [39]. Moreover, a significant increase in the protein abundance of AF9 was observed along with neural differentiation (Figure 1c), and co-immunostaining showed the specific localization of AF9 in TUJ1-positive immature neurons in differentiated cells at day 22 (Figure 1d). These findings implicate a role for AF9 in the regulation of hESC neural differentiation. 
To explore its function, $A F 9$ was knocked down in hESCs by lentivirus-mediated $A F 9$-specific shRNAs (Supplementary Figure S1B). Among infected cells, the percentages of $\mathrm{SOX}_{1}{ }^{+} \mathrm{NPs}$ at day $12, \mathrm{TUJ}^{+}$cells at day 22, and mature $\mathrm{NeuN}^{+}$neurons at day 32 significantly decreased (Supplementary Figure S1C and S1D; Figure 1e and f). Given the low AF9 expression in hESCs (Figure 1c), depletion of AF9 did not affect its
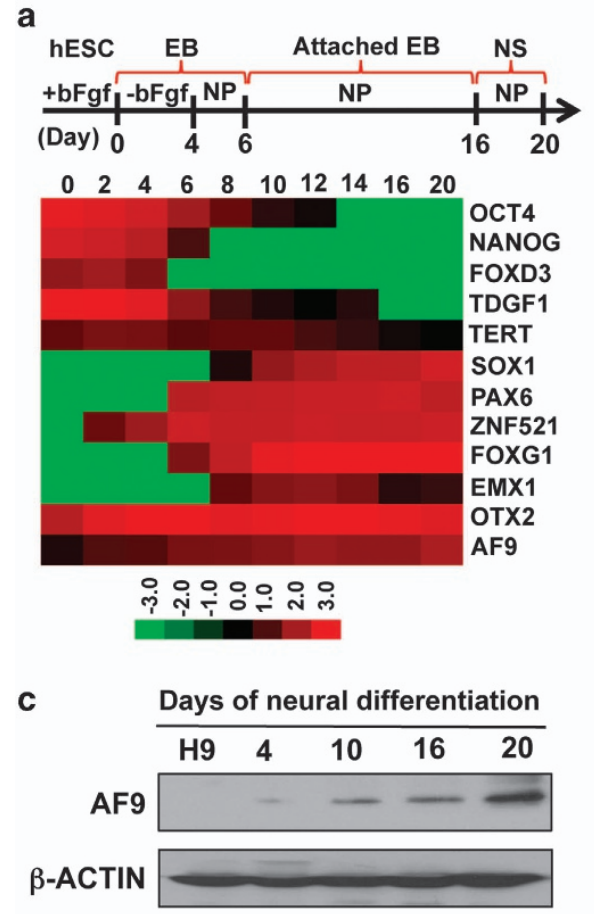

e

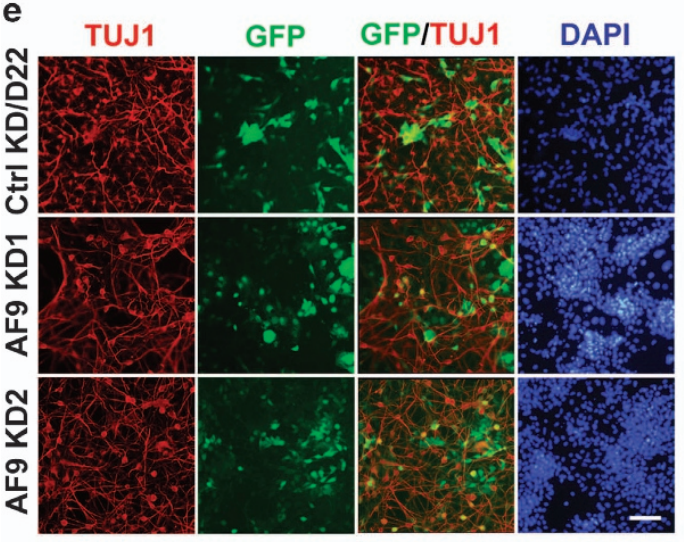

g

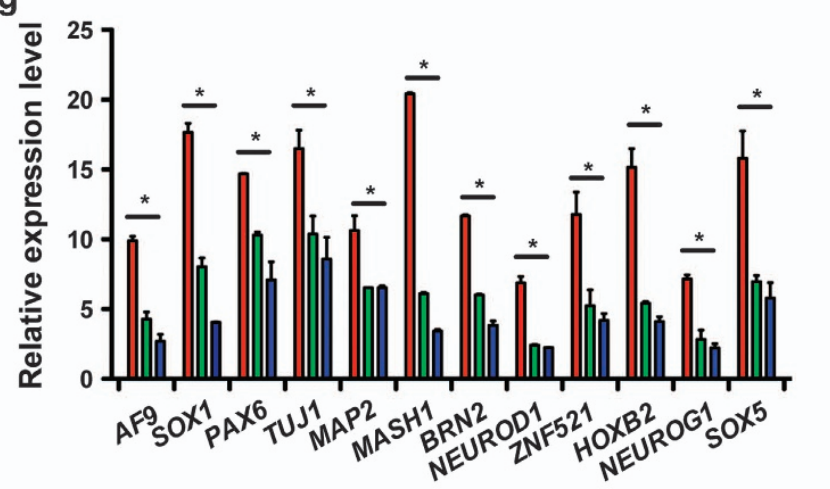

b
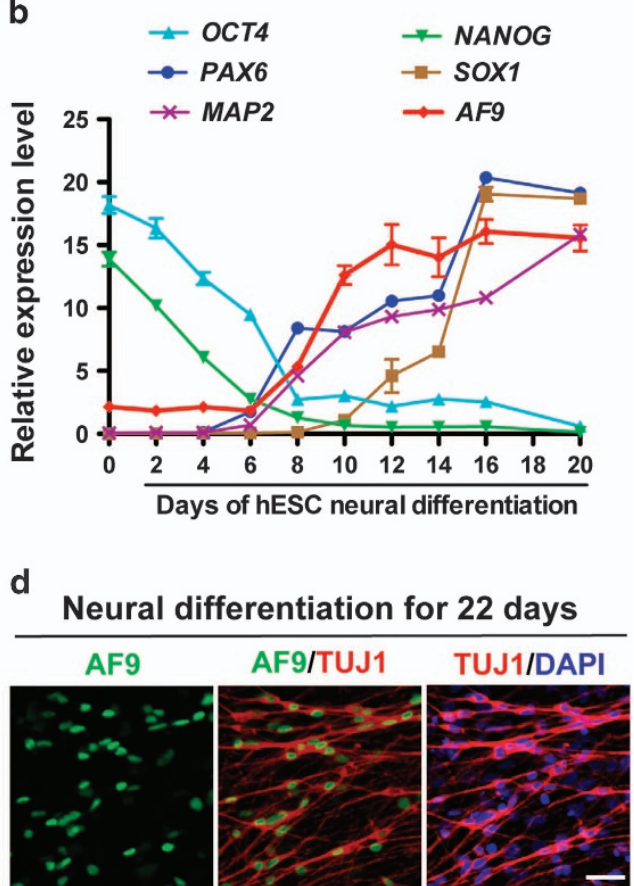

f

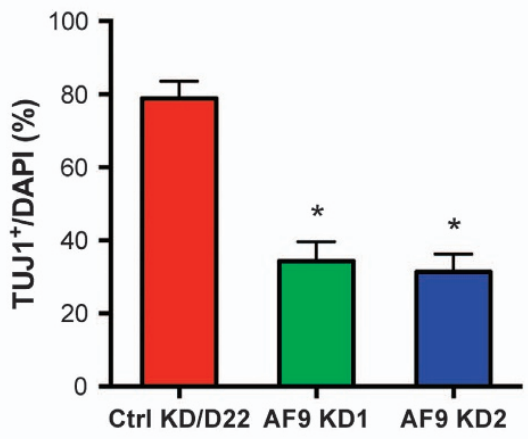

Ctrl KD1 KD2

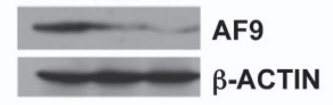


pluripotency (Supplementary Figure S1E). Moreover, AF9 knockdown does not affect the expression of other differentiating markers, which was maintained at a very low level when cells were differentiated in the neural-inducing environment (Supplementary Figure $\mathrm{S} 1 \mathrm{~F}$ ), whereas the expression of multiple neurodevelopmental genes was reduced in the $A F 9$-knockdown cells (Figure 1g). Clearly, disruption of neural differentiation program by $A F 9$ depletion suggests that AF9 is partially required for the acquisition of neural fate from pluripotent hESCs.

\section{AF9 overexpression promotes $h E S C$ neural commitment}

To address whether AF9 is sufficient in promoting hESC neural differentiation, $A F 9$ was cloned into a doxycycline (DOX)-inducible lentiviral expression vector containing an RFP reporter. To keep the DOX induction system stable and to make the cell counting easier, inducible AF9 lentivirus-infected hESCs were subjected to neural differentiation according to the modified protocol described in Figure 2A. In brief, lentivirus-infected hESCs were induced in the culture medium without bFGF for 8 days and in NP medium for another 2 days as embryonic bodies (EBs). Then, these EBs were attached for 2 days and subjected to immunostaining analysis with a TUJ1 antibody. AF9 overexpression was induced by DOX throughout the neural differentiation process. Comparing with the control group, overexpression of AF9 markedly enhanced the neural differentiation of hESCs with neurite outgrowth (Figure $2 \mathrm{Aa}-\mathrm{d}$ ). In day 12 differentiated cells, many $\mathrm{TUJ}^{+}$neurons were observed in the AF9-overexpressed cells in the marginal regions of the attached EBs, in which the neuronal marker TUJ1 was colocalized with RFP reporter reflecting AF9 expression (Figure 2Ae-i; Supplementary Figure S2A). The cells in the central region of the attached AF9-expressing EBs were mainly $\mathrm{MASH}^{+}$and a minority of these cells had differentiated into TUJ1 ${ }^{+}$ neurons (Supplementary Figure S2A and S2B). Furthermore, partial AF9-induced neurons that migrated from EBs had differentiated into $\mathrm{MAP}^{+}$and $\mathrm{NeuN}^{+}$neurons (Supplementary Figure S2C and S2D). These data suggest that AF9 overexpression accelerates the neural commitment of hESCs.

Next, we performed microarray analysis of lentivirus-infected cells at day 12 to identify AF9activated neural genes. In total, 2754 differentially expressed genes (1 669 upregulated and 1085 downregulated genes) were identified. Among the topranking upregulated genes in AF9-overexpressing cells were a large number of neurodevelopmental genes (Figure 2B). The expression of some altered genes was confirmed by qPCR in the AF9-overexpressing cells compared with control cells. Consistent with the microarray data, the transcriptional levels of $S O X 1 / 5$, PAX6, HOXB2, MASH1, NGN2, BRN2, NEUROD1, $T U J 1$, and $M A P 2$ were significantly upregulated by AF9 overexpression (Figure $2 \mathrm{C}$ and D). Interestingly, some hindbrain markers (HOXA2 and $H O X B 2)$ were strongly induced by AF9, whereas the expression of forebrain markers (FOXG1 and $G B X 2)$ was inhibited by AF9 overexpression (Figure 2B; Supplementary Figure S2E), suggesting that AF9-induced neural cells may possess hindbrain-like character and that AF9 might have a role in anterior-to-posterior patterning of the CNS. However, the expression of other germ layer markers was not affected by AF9 overexpression under the neural differentiation conditions (Supplementary Figure S2F).

Figure 1 AF9 is required for human embryonic stem cells (hESCs) neural differentiation. (a) The upper panel shows the diagram of hESC neural differentiation procedure used in this study (see detail in experimental procedures). The lower panel shows a heat map of pluripotency genes (OCT4, TDGF1, NANOG, FOXD3, and TERT), early neural genes (SOX1, PAX6, and ZFN521), forebrain markers (FOXG1, EMX1, and OTX2), and AF9 expression during hESC neural differentiation from day 0 to day 20 . The log transformed $^{2}$ normalized FPKM (fragments per kilo base per million) is assigned as expression values. (b) q-PCR analysis of OCT4, NANOG, SOX1, $P A X 6, M A P 2$, and AF9 during hESC neural differentiation. (c) Western blot analysis of AF9 protein levels showing the gradual upregulation of AF9 during hESC neural differentiation. $\beta$-Actin served as a loading control. (d) Immunostaining of AF9 (green) and the immature neuronal marker TUJ1 (red) with their specific antibodies in day 22 differentiated hESCs. DAPI staining is shown in blue. These results show the co-localization of AF9 and TUJ1 in neuronal cells. Bar, $50 \mu \mathrm{m}$. (e) hESCs were transfected with lentivirus-mediated control shRNA (Ctrl) or AF9 shRNAs (AF9 KD1 or AF9 KD2). The three established cell lines were subjected to neural differentiation for 22 days (D22) according to the protocol described in (a) and these cells were stained with an anti-TUJ1 antibody. The lentiviral vector expresses green fluorescent protein (GFP) to indicate the shRNA expression status. Bar, $50 \mu m$. (f) The percentage of TUJ1-positive cells was measured in control and AF9-knockdown cells. (g) The differentiated day 12 (D12) cells expressing control or AF9 shRNAs were collected for western blot analysis with an anti-AF9 polyclonal antibody and q-PCR analysis of the expression of AF9 and neurodevelopmental genes. 


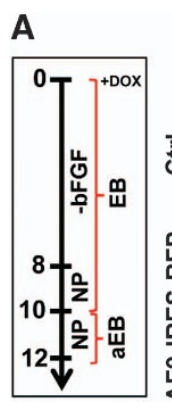

B

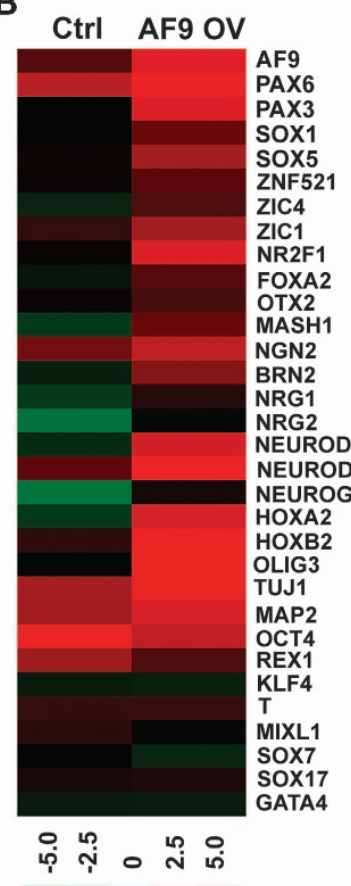

BF

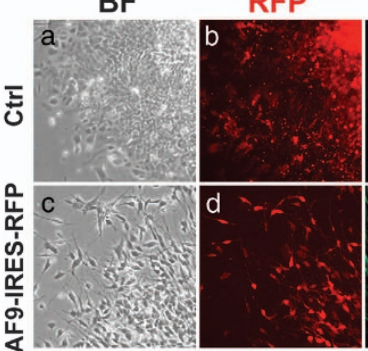

C
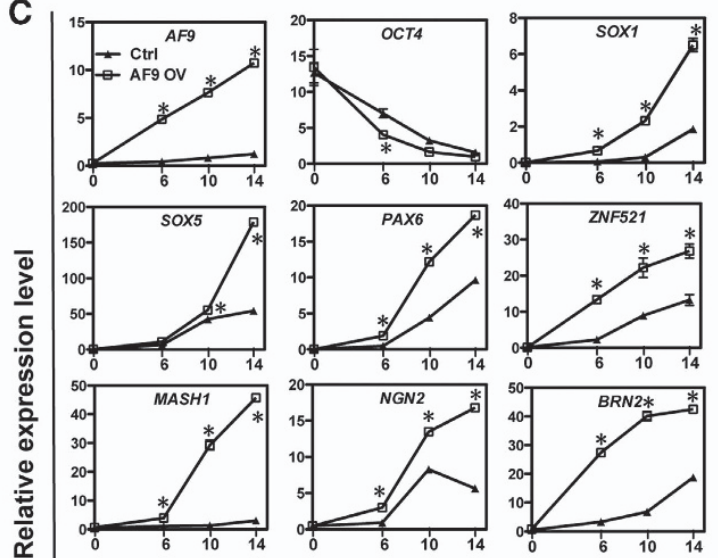

๘
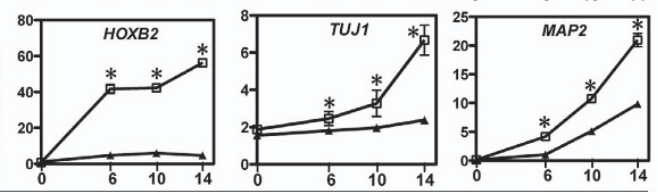

Days of neural differentiation

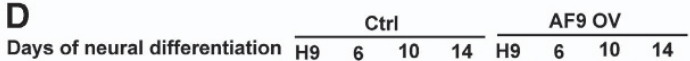

AF9

$\beta$-ACTIN
DAPI

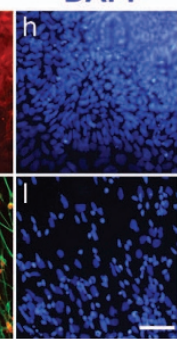


AF9 purification

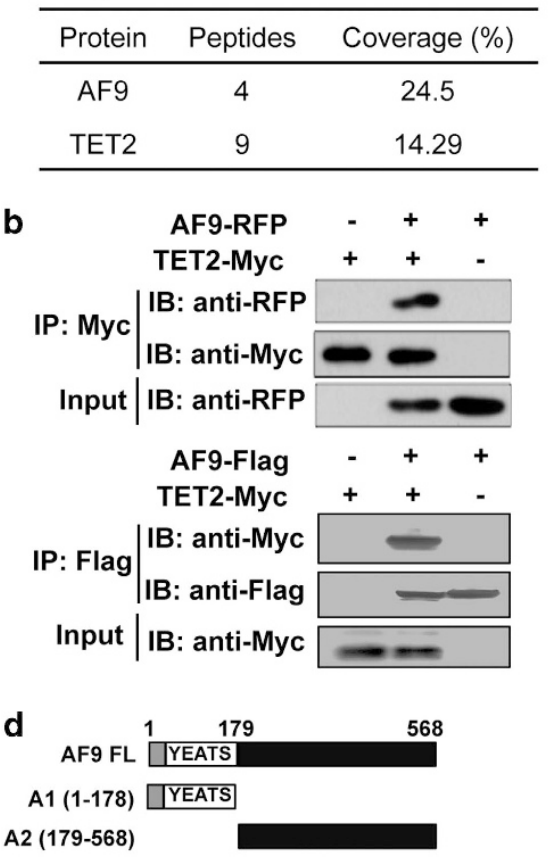

c

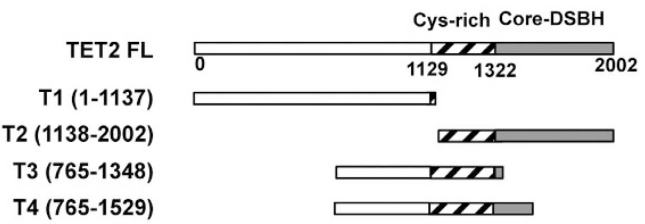

$\begin{array}{lllll}\text { FL } & \text { T1 } & \text { T2 } & \text { T3 } & \text { T4 }\end{array}$

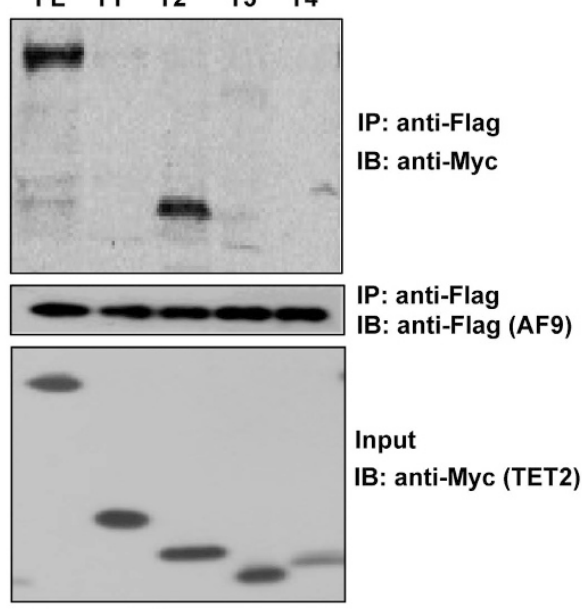

$\begin{array}{lll}\text { FL } & \text { A1 } & \text { A2 }\end{array}$

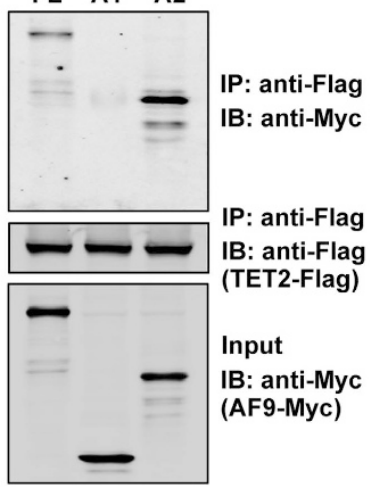

e

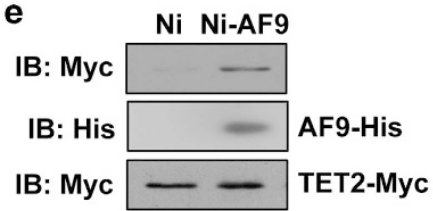

f

IP: anti-AF9

IP: IgG

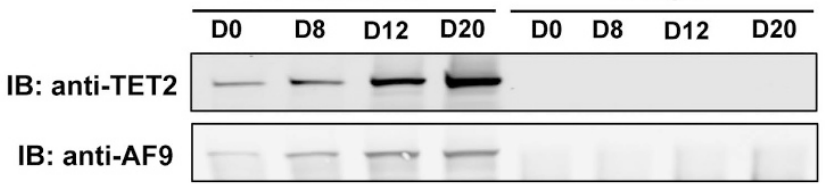

IP: $\lg G$

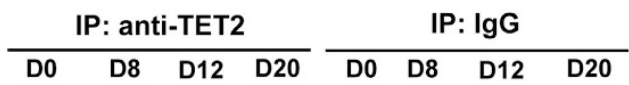

IB: anti-AF9

IB: anti-TET2

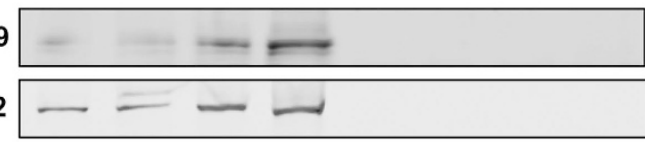

IB: anti-AF9

IB: anti-TET2
Input

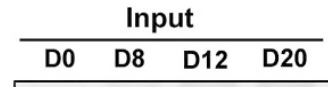

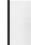

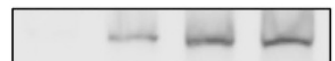

Figure 3 AF9 physically interacts with TET2. (a) Purification of AF9-associated proteins from day 12 differentiated human embryonic stem cells (hESCs). AF9-associated proteins were identified by mass spectrometry and are listed in the Supplementary Table S1. (b) TET2 interacts with AF9. AF9-RFP or AF9-Flag was co-expressed with TET2-myc in HEK293T cells and co-immunoprecipitations were performed with the indicated antibodies. Whole-cell lysate was used as an input. (c) AF9 interacts with the TET2 C-terminal domain. TET2 mutant constructs and AF9 were co-expressed in HEK293T cells. The T2 mutant containing the core-DSBH domain (catalytic domain) interacts with full-length AF9. (d) The C-terminal domain of AF9 interacts with TET2. Deletion mutants of AF9 mutants and full-length TET2 were co-expressed in HEK293T cells. The A2 mutant containing the C-terminal domain of AF9 interacts with TET2. (e) Half in vitro interaction between AF9 and TET2. His-tagged AF9 was purified from E. coli and incubated with cell lysate from HEK293T cells expressing TET2-Myc. (f) AF9 interacts with TET2 in differentiated hESCs. Cells were collected on Day 0, Day8, Day12, and Day20, respectively. IgG was used as an immunoprecipitation control; Rabbit polyclonal antibody against AF9 was used for the co-immunoprecipitation of TET2. 
In our microarray analysis, multiple neurodevelopmental genes including NP and neuronal genes were increased by AF9 overexpression. Given the sequential activation of neurodevelopmental genes during conventional hESC neural differentiation (Figure 1b; Supplementary Figure S1A), the time windows of neural gene activation by AF9 were assessed by controlling the time period of DOX treatment. The early neural markers ( $P A X 6$ and $S O X 1$ ) were more sensitive to induction by AF9 overexpression at the early stage of hESC neural differentiation, even though DOXinduced $A F 9$ transcription was reduced to the normal differentiating level at day 10 (Figure 2E). Conversely, DOX-induced AF9 at the later stage preferentially activated the proneural determinant $M A S H I$ and neuronal MAP2 genes (Figure 2E). Taken together, AF9 may accelerate hESC neural commitment through activating multiple neurodevelopmental genes during hESC neural commitment.

\section{AF9 physically interacts with TET2}

To identify the functional partner of AF9 in hESC neural differentiation, protein affinity purification was performed using an anti-AF9 antibody in day 12 differentiated cells, and the purified proteins were separated by sodium dodecyl sulfate-polyacrylamide gel electrophoresis (SDS-PAGE) (Supplementary Figure S3A). Mass spectrometry analysis identified 22 possible proteins including known AF9 partners, such as AF4 and HSP70 [20, 40], in the AF9-associated complex (Supplementary Table S1). AF9 modulates transcriptional activity through interacting with epigenetic regulators $[19,21]$. Intriguingly, the DNA dioxygenase TET2 was present in AF9-associated complex (Figure 3a). To confirm this interaction, we performed co-immunoprecipitation assays with overexpressed AF9 and TET2 or TET1 with different fusion tags in HEK293T cells. The results showed that both TET2 and TET1 were associated with AF9 (Figure 3b; Supplementary Figure S3B).

To map the interaction regions between AF9 and TET2, we generated four TET2 deletion mutants (T1-4) and two AF9-deletion mutants (A1-2) based on their tertiary structures. We found that the carboxyterminal catalytic domain of TET2 (TET2CD, T2) interacts with AF9 (Figure 3c) and that the carboxyterminal domain (A2), but not the YEATS domain (A1), of AF9 was required for the interaction with TET2 (Figure 3d). Furthermore, recombinant AF9His was purified from E. coli using nickel column chromatography, and TET2-containing lysate was obtained from TET2-myc-transfected HEK293T cells.
A pull down assay revealed that TET2-myc could be co-purified from cell lysates using recombinant AF9, suggesting that AF9 tightly associates with TET2 in vitro (Figure $3 \mathrm{e}$ ). To study the endogenous interaction, immunoprecipitation was performed using antiAF9 or anti-TET2 antibodies in differentiated cells and hESCs. AF9 and TET2 exhibited a specific interaction in differentiated neural cells but not hESCs, and they showed increasing interaction strength along with hESC neural differentiation, accompanied by the increasing protein levels of AF9 and TET2 (Figure 3f). Interestingly, point mutations in the $\mathrm{HxD}$ motif (H1382Y and D1384A) of TET2 that are catalytically inactive [41] disrupted the AF9-TET2 interaction (Supplementary Figure S3C). Together, these data demonstrate that AF9 physically interacts with TET2 both in vitro and in vivo.

\section{AF9 and TET2 cooperate to promote hESC neural differentiation}

Given that AF9 and TET2 may form a functional complex and AF9 is required for hESC neural differentiation, we asked whether TET2 is also involved in this process. Although TET1 was also shown to interact with AF9 by co-immunoprecipitation assays in HEK293T cells (Supplementary Figure S3B), only TET2 transcription was upregulated during hESC neural differentiation (Figure 4a), which is similar to the expression pattern of $A F 9$ (Figure 1b). To establish a functional connection between TET2 and neural differentiation, co-immunofluorescence was performed for TET2 and TUJ1 in day 22 cells; TET2 and TUJ1 were co-expressed in differentiated neuronal cells (Figure 4b), suggesting that TET2 might be a positive modulator of the neural differentiation process. Intriguingly, a majority of the $\mathrm{TET} 2^{+}$cells were $\mathrm{TUJ} 1^{+}$ neurons induced by AF9 overexpression (Figure 4c). The activation of TET 2 expression may parallel the AF9-induced progression of neural differentiation, and AF9 functions might correlate with TET2 actions.

Lentivirus-mediated knockdown assays were performed to investigate TET2 function in hESC neural commitment. Similar to AF9, TET2 knockdown resulted in a decreased proportion of $\mathrm{TUJ}^{+}{ }^{+}$neurons (Figurs $4 \mathrm{~d}$ and e) and downregulation of multiple neural genes, such as SOX1, ZNF521, MASH1, $H O X B 2$, and MAP2 (Figure 4f). In contrast, TET2 knockdown had no significant effect on the generation of other cell lineages (Supplementary Figure S4A). Moreover, depletion of TET1 by its specific shRNA generated nearly no effect on neural differentiation (Supplementary Figure S4B), suggesting that TET1 is 
not the functional partner of AF9 in hESC neural commitment processes. These results demonstrate that TET2 partially contributes to human neural gene activation and hESC neural differentiation.
AF9 cooperates with distinct epigenetic factors to participate in epigenetic modifications [14, 22, 23]; we therefore asked whether AF9 and TET2 cooperatively participate in hESC neural differentiation. To test this, a

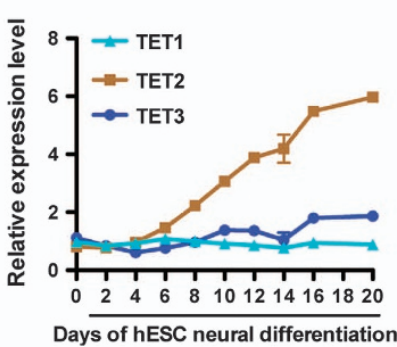

C

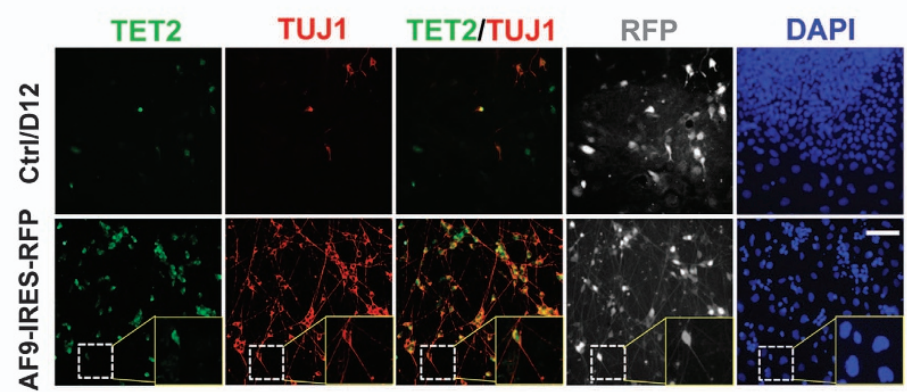

b

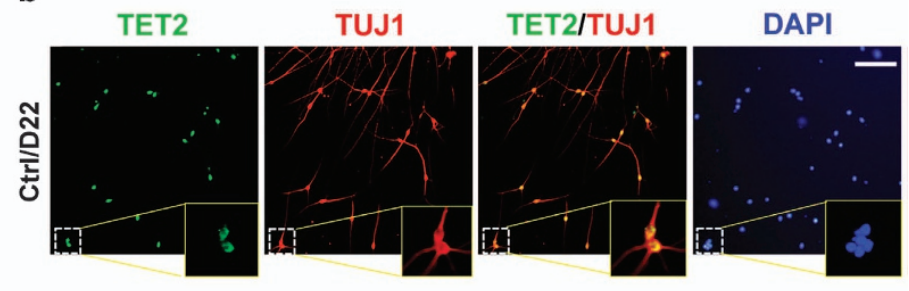

e

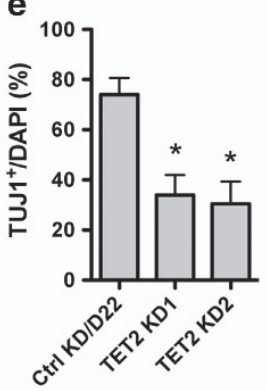

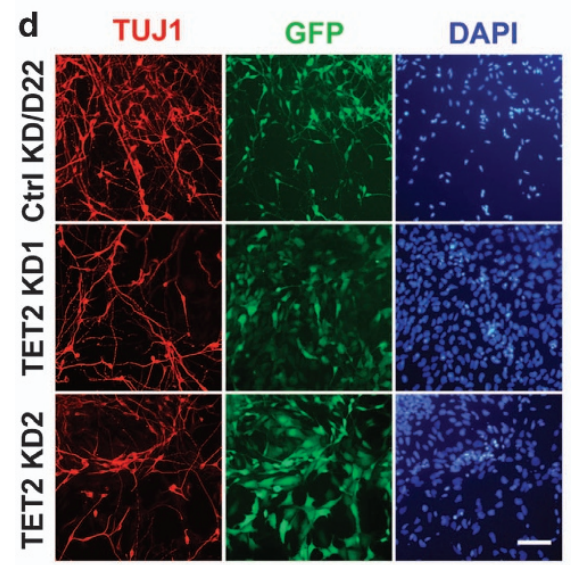

g

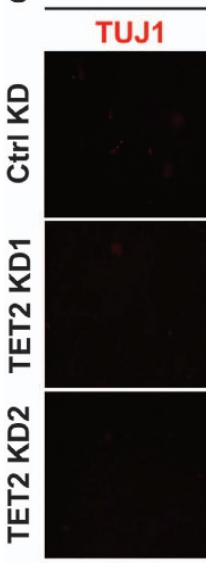

Ctrl/D12 GFP f
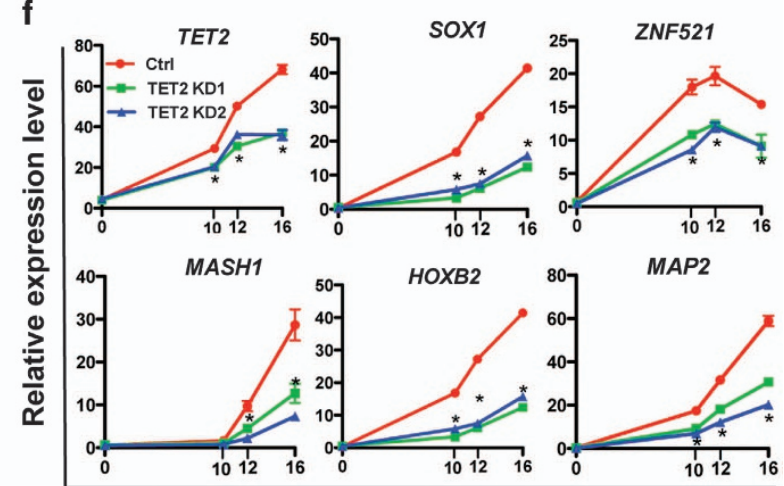

Days of neural differentiation

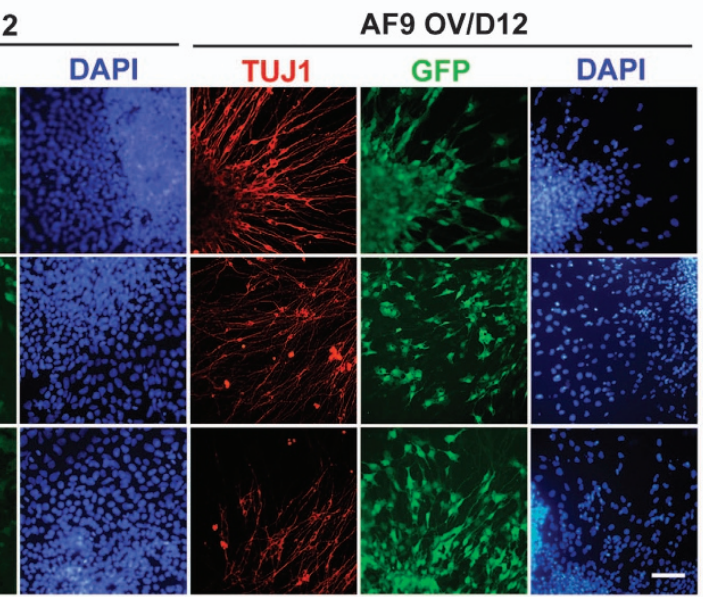

h

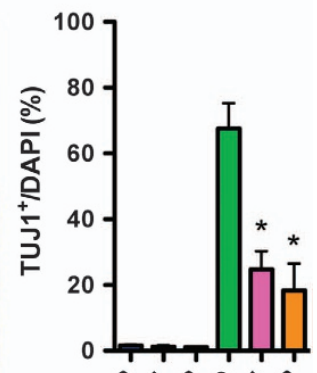

$s^{2}+0^{2}+80^{2}+0^{2}$

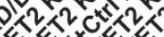

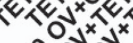

सिद्ध 
control or AF9-overexpressing hESCs were co-infected with TET2-specific shRNAs or control shRNA. The established hESC lines were subjected to neural differentiation for 12 days. We found that very few $\mathrm{TUJ} 1^{+}$cells were derived from control cells either co-expressing control shRNA or TET2 shRNAs. However, for the AF9-overexpression group, the percentage of $\mathrm{TUJ}^{+}$cells was much lower in the TET2-knockdown cells $(<30 \%)$ than in the control knockdown cells $(\sim 70 \%)$ (Figure $4 \mathrm{~g}$ and $\mathrm{h}$ ). Consistently, the expression level of key neural regulators (SOX5, NEUROG1, HOXB2, MASH1, and MAP2) was decreased by TET2 knockdown in both the control and AF9-overexpressing cells (Supplementary Figure S4C), confirming the observation that AF9-induced hESC neural differentiation as impaired by TET2 depletion. Moreover, TET2 overexpression could further enhance the neural promoting activity of AF9 (Supplementary Figure S4D), and the neural promoting effect of TET2 was disrupted by catalytic negative mutations (Supplementary Figure S4E). Together, these data suggest that AF9 and TET2 cooperate to promote hESC neural commitment.

\section{AF9 and TET2 are enriched at the common target loci of key neural genes}

The above findings indicated that AF9 and TET2 could cooperatively regulate key neural gene expression in hESC neural differentiation, while it remained unknown whether the AF9-TET2 complex directly regulated these neural genes. To address this question, the genome-wide distribution of AF9 and TET2 on chromatin of human neural cells was determined by chromatin immunoprecipitation sequencing analysis (ChIP-seq) using anti-AF9 and anti-TET2 antibodies, the binding specificity of which was predetermined with good yield (Supplementary Figure S5A and S5B).
AF9 displayed similar binding profiles to TET2, and their binding sites are mainly located around the transcriptional start sites (TSSs) of target genes or in distal regions $(>50 \mathrm{~kb}$ ) (Figure 5a). Gene ontology analysis revealed that both AF9 and TET2 were positively involved in the regulation of neural-related cellular processes, such as neurogenesis, axonogenesis, and synapse formation (Supplementary Figure S5C). Moreover, AF9 and TET2 target genes overlapped considerably (Figure 5a), and 48 of these genes were affected by AF9 overexpression (Figure 5b).

The 35 co-occupied and AF9-activated genes included multiple key neural regulators (SOX5, MASH1, ZNF521, MAP2, NEUROG1, and HOXB1-3). AF9 and TET2 co-occupied the DNA regions around the TSSs of these genes with one (R1) or two (R1 and R2) overlapping binding regions (Figure 5c; Supplementary Figure S5D). Most occupied regions at common target genes were associated with relatively high GC density (Figure 5c). Combining the expression patterns upon AF9 overexpression, ZNF521 and HOXB2 were strongly enhanced at day 6 (Figure 2c), demonstrating that ZNF521 and HOXB2 are early AF9 target genes, while other targets might be activated at later stages. Furthermore, the essential neural regulators $P A X 3 / 7$ and $S O X-O T$, a long non-coding RNA specifically expressed in mouse brain [42], were also co-occupied by AF9 and TET2 (Supplementary Figure S5E). Interestingly, AF9 also bound to the TET2 promoter regions (Supplementary Figure S5E), suggesting that AF9 might be an upstream factor of TET2. Furthermore, de novo motif discovery analyses were conducted to identify AF9 and TET2 binding motifs within their bound regions. Among the top ranking motifs for AF9, consensus AAC-containing elements were discovered (Figure 5d, left). Besides, C-rich sequences were found in the TET2-bound regions

Figure 4 AF9 and TET2 cooperatively promote human embryonic stem cell (hESC) neural differentiation. (a) TET1, TET2, and TET3 mRNA levels were determined by q-PCR during hESC neural differentiation. TET1 and TET3 were maintained at stable levels, whereas TET2 expression gradually increased during hESC neural commitment. (b) Neural-differentiated cells at day 22 (D22) were co-immunostained with anti-TET2 and anti-TUJ1 antibodies. The result shows that TET2 is mainly expressed in TUJ1-positive neurons. (c) Control (Ctrl) or AF9-overexpressing (AF9-IRES-RFP) cells were differentiated for 12 days according to the protocol in Figure 2A and co-stained with anti-TET2 and anti-TUJ1 antibodies. AF9 overexpression-induced neurons (TUJ1 positive) were also TET2 positive. (d) In vitro neural differentiation of hESCs infected with lentiviral control shRNA (Ctrl) or TET2 shRNAs (TET2 KD1 or TET2 KD2) and cultured for 22 days according to the protocol described in Figure 1a. The cells were stained with an anti-TUJ1 antibody. Green fluorescent protein (GFP) served as a marker for shRNA expression. (e) The percentages of TUJ1-positive cells were calculated in the control and TET2-knockdown cell populations. (f) The relative expression of TET2, SOX1, MASH1, NEUROD2, HOXB2, and MAP2 was examined by q-PCR in control shRNA- (Ctrl) or TET2 shRNA-expressing hESCs undergoing neural differentiation. (g) Vector (Ctrl) or AF9-overexpressing (AF9 OV) hESCs were co-infected with lentiviral control shRNA (Ctrl KD) or TET2 shRNAs. These cells were subjected to neural differentiation for 22 days and then immunostained with an anti-TUJ1 antibody. Bars, $100 \mu \mathrm{m}$. (h) The percentages of TUJ1-positive cells in $(\mathbf{g})$. 

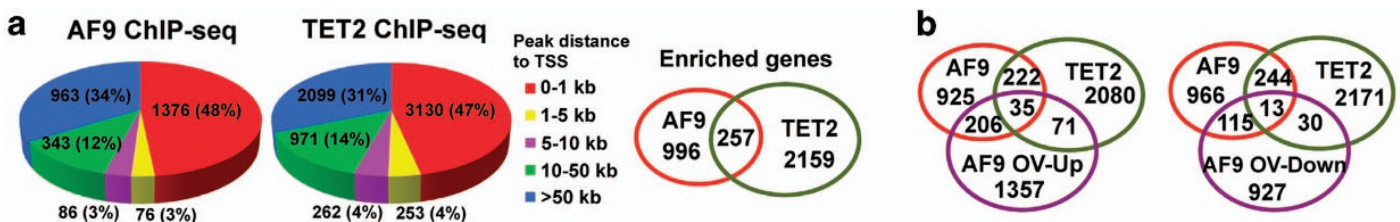

Coverlapping binding region 1

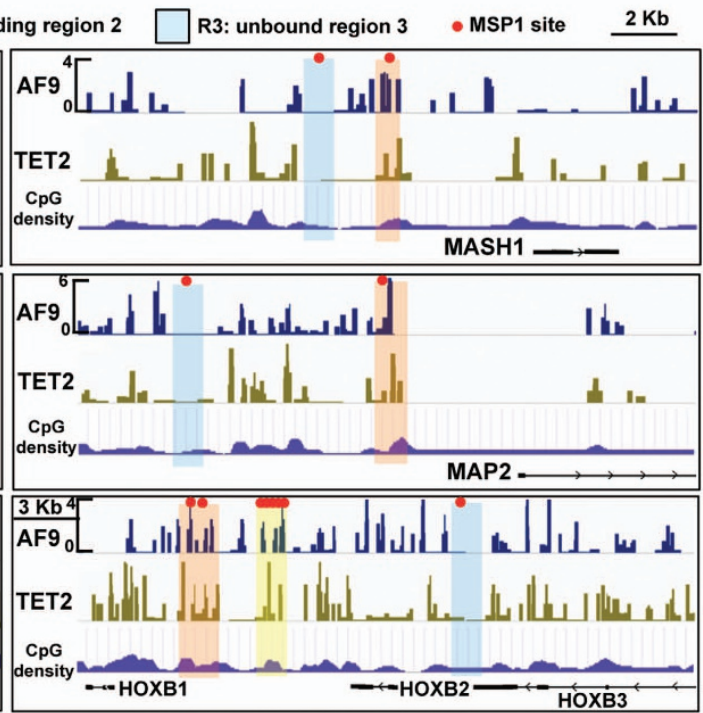

d

\begin{tabular}{|r|c|}
\hline AF9 binding motifs & P-value \\
\hline ATAACIACCAA & $1 \mathrm{E}-14$ \\
GGTCAACAACAT & $1 \mathrm{E}-13$ \\
TGAAAAGAAC & $1 \mathrm{E}-12$ \\
\hline
\end{tabular}

\begin{tabular}{|c|c|}
\hline TET2 binding motifs & P-value \\
\hline GICCTCCCCACC & $1 \mathrm{E}-16$ \\
ACA CCCT $\mathrm{AT}$ & $1 \mathrm{E}-10$ \\
CTGCCCCCCG & $1 \mathrm{E}-9$ \\
\hline
\end{tabular}
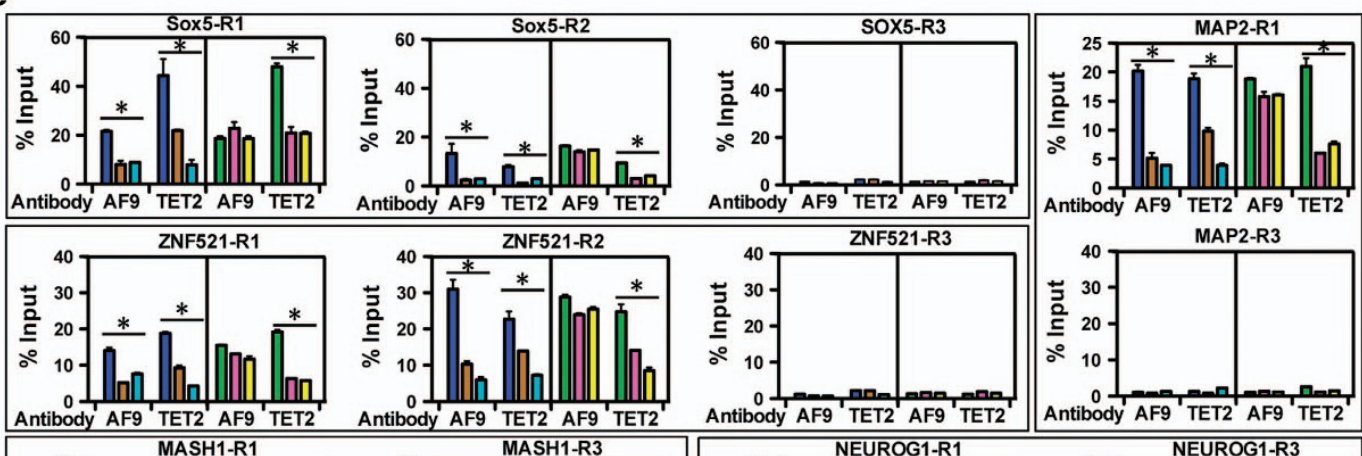

Antibody AF9 TET2 AF9 TET2

Antibody AF9 TET2 AF9 TET2
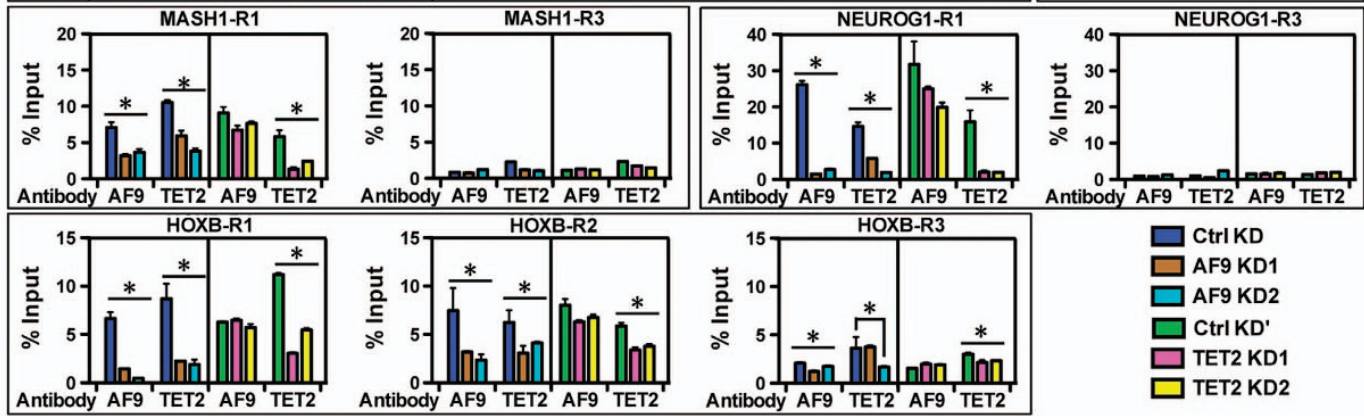

Antibody AF9 TET2 AF9 'TET2

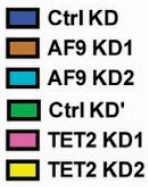


(Figure 5d, right) that were close to AF9-recognizing elements. Indeed, these adjacent motifs were present at the binding sites of most AF9-TET2 common target genes. Comparably, AF9-recognizing elements were much more specific than that of TET2-bound DNA sequences.

Next we validated the ChIP-seq results by using ChIP-qPCR to examine different loci in day 12 AF9and TET2-knockdown cells (Figure 5e). In control knockdown cells, AF9 and TET2 showed strong binding activity at the $\mathrm{R} 1$ and/or $\mathrm{R} 2$ binding sites of the tested neural target genes. Remarkably, AF9 and TET2 binding activities in co-occupied regions were specifically decreased by $A F 9$ or $T E T 2$ knockdown, respectively. As negative control sites, it was hard to detect the binding signals in the $\mathrm{R} 3$ regions (Figure 5e). More importantly, the TET2 binding activity at neural target gene loci was markedly decreased in the AF9-knockdown cells (Figure 5e), suggesting that AF9 depletion probably suppressed the recruitment of TET2 to these loci. In contrast, knockdown of TET2 did not disturb the recruitment of AF9 to the same loci (Figure 5e). Most likely, AF9 binding occurs before TET2 occupancy at the common loci, which is dependent on AF9 recruitment. Moreover, AF9-mediated TET2 targeting correlated with the expression levels of target genes (Supplementary Figure S4B). Combining the identification of AF9-TET2 interacting complex and their recognizing elements (Figure 3, Figure 5d and e), our data suggest that AF9 guides TET2 to target loci and that AF9 and TET2 might be sequentially recruited onto the regulatory regions of common downstream targets to form a complex to modulate neural gene transcription.

\section{$5 \mathrm{mC}$ conversion to $5 \mathrm{hmC}$ at key neural gene loci during hESC neural differentiation}

We next asked how AF9-TET2 complex achieves the gene regulation. DNA methylation at gene promoters is inversely correlated with the expression of the associated genes. Tet 1 and Tet 2 have critical roles in $5 \mathrm{mC}$ oxidation to $5 \mathrm{hmC}$, inducing DNA demethylation in primordial germ cells [43, 44]. Single-base resolution $5 \mathrm{hmC}$ mapping revealed that $5 \mathrm{hmC}$ was enriched within active genomic regions in mouse and human brains [35]. As TET2 could regulate hESC neural differentiation (Figure 4) and TET proteins act through the regulation of DNA methylation status [29], we investigated whether $5 \mathrm{hmC}$ generation was associated with the activation of TET2 target genes during hESC neural differentiation.

The global $5 \mathrm{hmC}$ status in differentiated human neural cells was examined using co-immunostaining assays. It was found that $5 \mathrm{hmC}$-positive cells were co-labeled with TUJ1, TET2, and AF9 in day 22 differentiated cells (Figure 6a), suggesting that $5 \mathrm{hmC}$ might be correlated with hESC neural commitment. To confirm this, glucosyltransferase-qPCR (Glu-qPCR) [43], was performed to analyze the $5 \mathrm{hmC}$ enrichment at neural gene loci (R1-3, Figure 5c) at different time points. We found that the $5 \mathrm{hmC}$ levels at the $\mathrm{R} 1$ and R2 loci of AF9-TET2 common target genes ( $O X X 5$, ZNF521, MASH1, NEUROG1, MAP2, and HOXB) increased during neural differentiation, while $5 \mathrm{hmC}$ at the control R3 loci of assessed genes remained low throughout the process (Figure 6b).

Subsequently, we performed $\mathrm{mC} / \mathrm{hmC}$-DIP-seq analysis to determine the $5 \mathrm{mC}$ and $5 \mathrm{hmC}$ levels in hESCs and day 12 differentiated cells. The $5 \mathrm{mC}$ and $5 \mathrm{hmC}$ peaks were mainly located in the proximal (within $\pm 1 \mathrm{~kb}$ ) and distal $(> \pm 50 \mathrm{~kb}$ ) regions relative to the TSS (Figure 6c), which was consistent with the observed AF9 and TET2 distributions (Figure 5a). As expected, the number of $5 \mathrm{mC}$ peaks in hESCs was greater than that in day 12 cells, whereas day 12 cells possessed more $5 \mathrm{hmC}$ peaks than hESCs (Figure 6c). The genes, in which the enrichment of $5 \mathrm{mC} / 5 \mathrm{hmC}$ is identified within $\pm 5 \mathrm{~kb}$ regions flanking TSSs, were

Figure 5 AF9 and TET2 co-occupy in neurodevelopmental gene loci. (a) ChIP-seq analysis of AF9 and TET2 binding sites using anti-AF9 and anti-TET2 antibodies in day 12 cells differentiated from human embryonic stem cells (hESCs). The distributions and percentages of the binding peaks are shown according to the peak distance to transcription start sites (TSSs). The number of AF9 and TET2 co-occupied genes $(<5 \mathrm{~kb})$ is shown in the right panel. (b) The AF9-enriched and TET2-enriched genes according to ChIP-seq analysis were compared with upregulated genes (left panel, AF9 OV-Up) or downregulated genes (right panel, AF9 OV-Down) upon AF9 overexpression (AF9 OV); the number of genes in each overlapping set was counted. (c) The binding peaks of AF9 and TET2 in the promoter regions around the transcriptional start site (TSSs) of AF9 and TET2 co-occupied genes (SOX5, MASH1, ZNF521, NEUROG1, $M A P 2$, and $H O X B$ ) are shown. R1 and R2 represent the overlapping binding regions 1 and 2, and R3 represents the unbound region 3 . The red dots represent MSP1 enzymatic sites. The lengths of displayed genomic regions are $20 \mathrm{~kb}$, except for the displayed $H O X B$ genomic region, which is $30 \mathrm{~kb}$ long. (d) AF9 and TET2 binding motifs and the $P$-value for each motif. (e) ChIP-qPCR verification of AF9 and TET2 enrichment at the R1 and R2 regions of neural target genes. The R3 region served as a negative control. R1-R3 are shown in panel (c). 
a

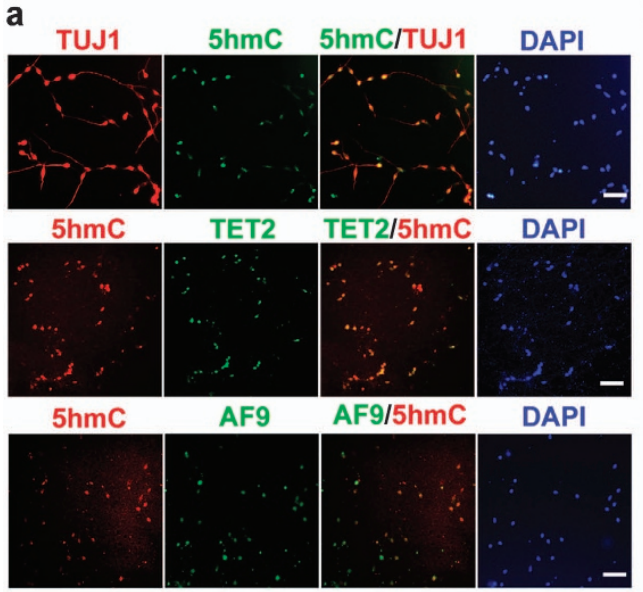

C

H9

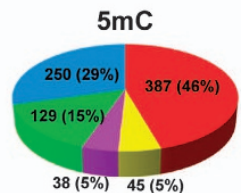

D12
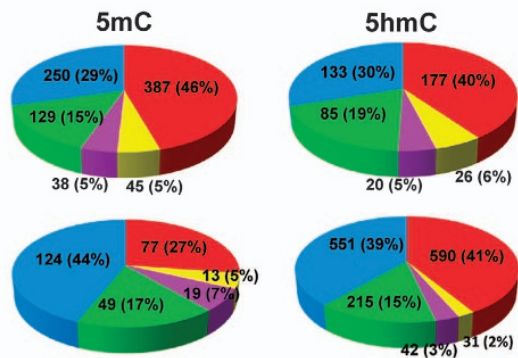

$0-1 \mathrm{~kb}$

$1-5 \mathrm{~kb}$

= 5-10 kb

$=10-50 \mathrm{~kb}$

- $>50 \mathrm{~kb}$
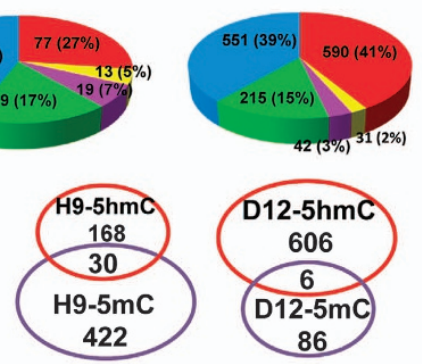

e
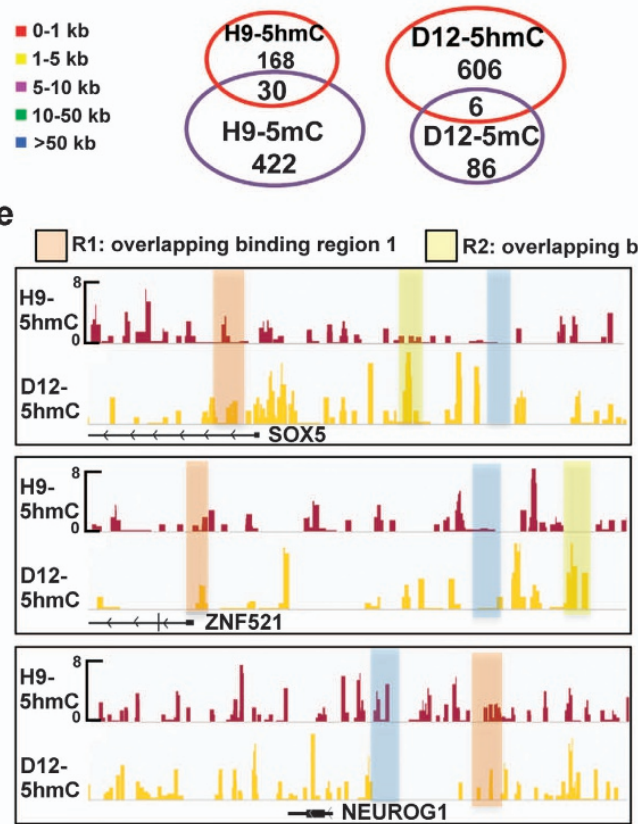

b
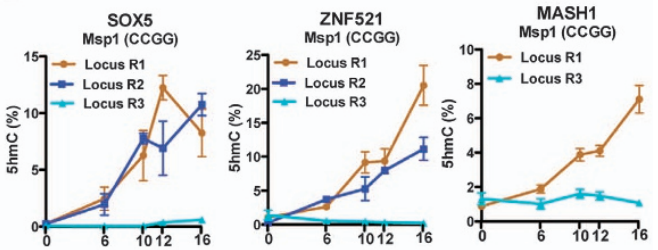

NEUROG1
Msp1 (CCGG)

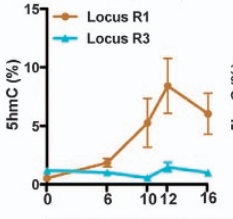

MAP2
Msp1 $(C C G G)$

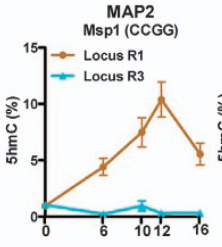

HOXB
Msp1 (CCGC)

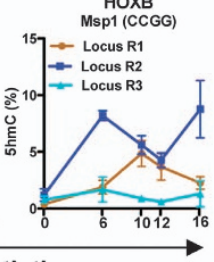

Days of neural differentiation

d

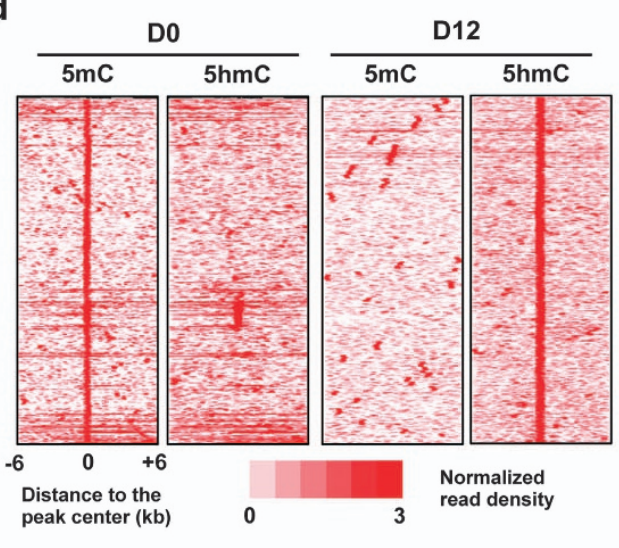

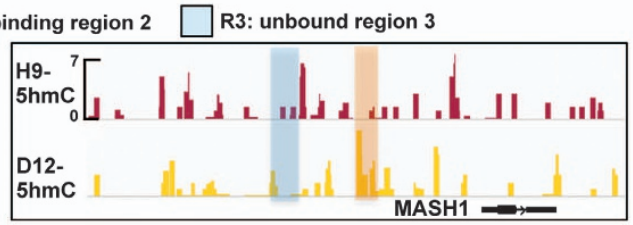
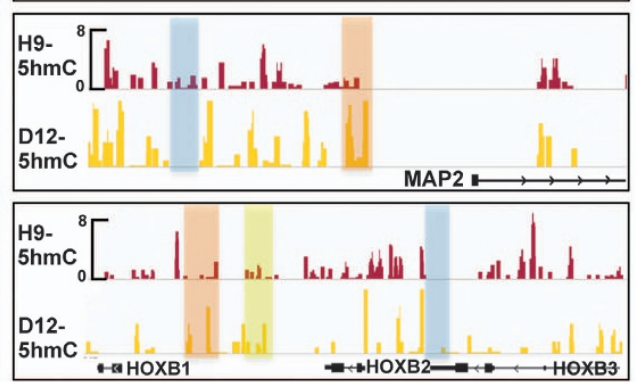

Figure $65 \mathrm{mC}$ conversion to $5 \mathrm{hmC}$ at neural gene loci during hESC neural differentiation. (a) The co-labeling of 5hmC with TUJ1, TET2, and AF9 in human neural cells. Cells were stained for $5 \mathrm{hmC}$ and co-stained for TUJ1, TET2, or AF9 in day 22 cells. Bars, $50 \mu \mathrm{m}$. (b) Glu-qPCR showing quantitative levels of 5hmC at MSP1 (CCGG) sites in the co-occupied regions of neural-related gene loci from day 0 to day 16. R1-R3 are shown as in Figure 5c. (c) Genome-wide distributions of $5 \mathrm{mC}$ and $5 \mathrm{hmC}$ were determined by MeDIP-seq and hMeDIP-seq. The peak distances to transcriptional start sites (TSSs) are shown. The genes that were enriched with peaks within $5 \mathrm{~kb}$ upstream or downstream of TSSs are defined as $5 \mathrm{mC}$ or $5 \mathrm{hmC}$ possibly regulated genes. The numbers of overlapped 5mC- and 5hmC- enriched genes $(P$-value $<0.00001)$ in $\mathrm{H} 9$ and D12 cells were counted. (d) The global distributions of $5 \mathrm{mC}$ and $5 \mathrm{hmC}$ in day 0 and day 12 cells. The normalized read density was assigned as $5 \mathrm{mC} / 5 \mathrm{hmC}$ levels within the regions flanking the center of $5 \mathrm{mC} / 5 \mathrm{hmC}$ peaks. (e) The $5 \mathrm{mC}$ and $5 \mathrm{hmC}$ distributions within the same genomic regions of key neural genes as shown in Figure $5 \mathrm{c}$. 
defined as possible $5 \mathrm{mC} / 5 \mathrm{hmC}$-related genes. During the fate determination from hESC to neural cells, the number of $5 \mathrm{mC}$-enriched genes decreased while the number of $5 \mathrm{hmC}$-enriched genes increased; only a few genes enriched for both $5 \mathrm{mC}$ and $5 \mathrm{hmC}$ were observed (Figure 6c). In addition, heatmaps of $5 \mathrm{mC} / 5 \mathrm{hmC}$ distributions (normalized read density) within the regions flanking the center of $5 \mathrm{mC} / 5 \mathrm{hmC}$ peaks were analyzed according to the previously reported method [45]. We found that $5 \mathrm{mC}$ peak density markedly decreased during neural conversion from hESCs, accompanied by the remarkable increase of $5 \mathrm{hmC}$ peak signals in the same genomic regions (Figure 6d). It suggests that $5 \mathrm{mC}$ in the 5mC-enriched DNA regions in hESCs was locally converted into $5 \mathrm{hmC}$ during hESC neural differentiation, leading to the loss of $5 \mathrm{mC}$ enrichment. The gene ontology terms of $5 \mathrm{hmC}$-enriched genes (active) in hESCs were highly similar to that of $5 \mathrm{mC}$-enriched genes (inactive) in day 12 cells, whereas the gene ontology terms of $5 \mathrm{hmC}$-enriched genes in day 12 cells were associated with neural morphogenesisrelated functions (Supplementary Figure S6). Next, the $5 \mathrm{hmC}$ enrichment on AF9-TET2 common neural target genes was examined. Rare $5 \mathrm{hmC}$ peaks were observed at the loci of the analyzed genes in hESCs, and there was no $5 \mathrm{hmC}$ enrichment at the control R3 regions in both hESCs and day 12 cells (Figure 6e). In contrast, $5 \mathrm{hmC}$ peaks were newly present coincidently at the R1/R2 loci or nearby regions, which were the common binding sites of AF9 and TET2, in day 12 neural cells (Figure 6e). Together, these results suggest that $5 \mathrm{mC}$ is efficiently converted to $5 \mathrm{hmC}$ at a large cohort of genomic regions during hESC neural differentiation.

The AF9-TET2 complex regulates $5 \mathrm{hmC}$ generation at neural gene loci

A study of TET2-knockout mice proposed that DNA demethylation at 5hmC-poised loci depends on TET2 activity [35]. In day 22 differentiated cells, $5 \mathrm{hmC}$ was co-localized with AF9 and TET2 proteins in $\mathrm{TUJ}^{+}{ }^{+}$neurons (Figure $6 \mathrm{a}$ ). Having demonstrated the cooperative effect of the AF9-TET2 complex in hESC neural commitment, we next addressed whether the AF9-TET2 complex modulated $5 \mathrm{hmC}$ generation at their common target gene loci. Therefore, the effect of AF9 overexpression on $5 \mathrm{hmC}$ generation was assessed by immunostaining. We found a large number of $5 \mathrm{hmC} / \mathrm{TUJ} 1$ double-positive cells after AF9 overexpression, but very few in the control experiment (Figure 7a). This result indicates that AF9 could promote $5 \mathrm{hmC}$ production in neural cells at the global level. At specific neural gene loci (SOX5, ZNF521, MASH1, NEUROG1, and MAP2), AF9 overexpression enhanced $5 \mathrm{hmC}$ levels while AF9 and TET2 depletion specifically decreased $5 \mathrm{hmC}$ distributions at the R1 and R2 regions. Moreover, AF9 overexpression-induced $5 \mathrm{hmC}$ increase was attenuated by TET 2 knockdown (Figure $7 \mathrm{~b}$ ). In contrast, $5 \mathrm{mC}$ levels at these neural gene loci, detected by DIP-qPCR assays using an anti-5mC antibody, were downregulated by AF9 overexpression and upregulated by AF9 or TET 2 knockdown, and the decrease of $5 \mathrm{mC}$ levels by AF9 overexpression was attenuated by TET2 depletion (Figure 7c). It demonstrates that the function of AF9-TET2 complex in 5mC-to-5hmC conversions at the neural gene loci depends on TET2 activity. We also found that the neural promoting effects by the overexpression of proneural gene MASH1 were partially attenuated by AF9 and TET2 double knockdown (Supplementary Figure S7), further indicating that AF9-TET2 complex accelerates hESC neural differentiation through activating their common downstream targets. Collectively, these data suggest that AF9 and TET2 regulate hESC neural differentiation through modulating $5 \mathrm{mC}$-to-5hmC conversions to activate key neural genes.

\section{Discussion}

Vertebrate neural development is coordinated by the integration of signaling pathways, key neurodevelopmental factors, and epigenetic marks. Epigenetic regulation of gene transcription is an essential and intrinsic mechanism for developmental assurance [13]. Using hESC neural differentiation as a model for human neural development, we discovered that the epigenetic factors AF9 and TET2 regulate neural development through forming a complex to directly activate the expression of key neurodevelopmental genes via an epigenetic mechanism. Similar to the function of mouse Af9 in embryonic patterning and neuron development $[15,16]$, human AF9 is required for hESC neural commitment through transcriptional regulation of master neural genes (Figures 1, 2 and 5). AF9 is known to modulate histone methylation by interacting with DOT1A or DOT1L [22, 46], and MLL-AF9 protects against DNA methylation of specific $\mathrm{CpG}$ nucleotides at the HOXA9 gene promoter [24]. These findings suggest that AF9 may act as a co-factor to coordinate the enzymatic activities of other proteins in epigenetic modifications. Our mechanistic study identifies AF9 as an active regulator of DNA hydroxylation, through interacting with TET2 and 
guiding TET2 to specific neural gene loci in neurodevelopment, resulting in the $5 \mathrm{mC}$-to- $5 \mathrm{hmC}$ conversions and neural gene activation. Although Af9 has been implicated in cortical development through mediating H3K79 dimethylation, the functional effect of Af9 in this context exclusively relies on its associated
partner-Dot1L [14], which was expressed in both hESCs and NPCs at stable levels (data not shown) and was not detectable in AF9-associated complex (Supplementary Table S1). It suggests that AF9mediated histone methylation might not be involved here and that TET2 is the specific functional partner of

a
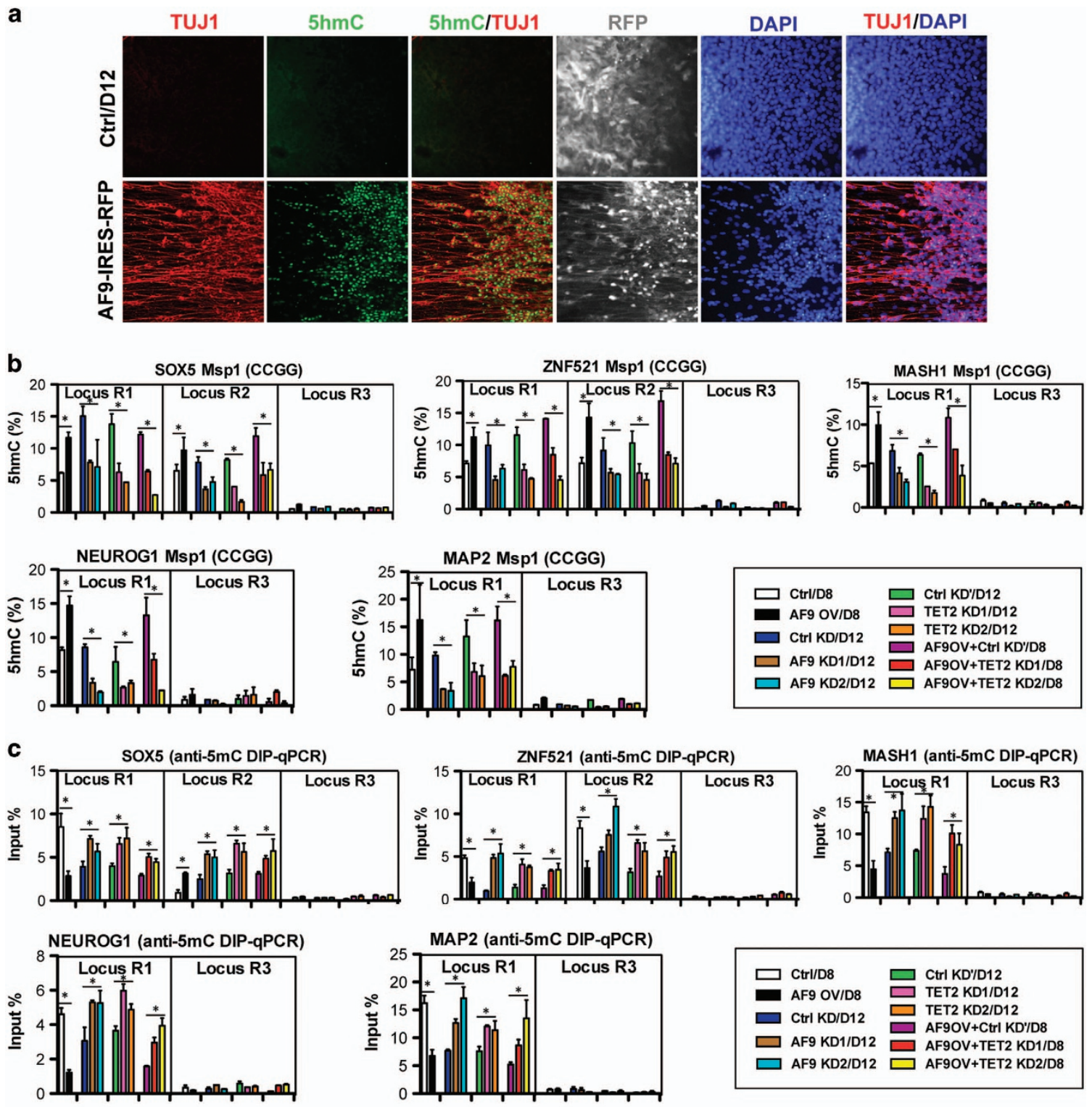

d

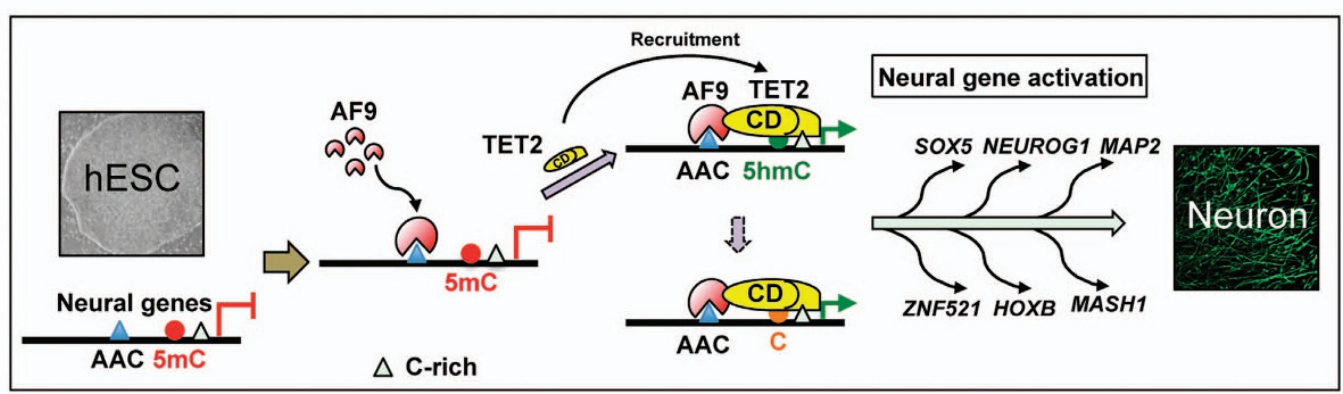


AF9 in hESC neural differentiation through modulating DNA hydroxylation.

TET family proteins and their catalyzed product $5 \mathrm{hmC}$ have been extensively associated with neurodevelopment. Xenopus Tet3 directly activates master neural genes ( $r x$, pax6, and ngn2) in eye and neural development during embryogenesis [38]. Murine Tet1 regulates hippocampal neurogenesis and lineage specification, and promotes active DNA demethylation in the adult brain and mouse ESCs (mESC) [30, 31, $47,48]$. Recently, it has been reported that mouse Tet3 is important for mESC neural differentiation [49]. Although Tet1- and Tet2-knockout mice develop normally [50, 51], Tet3 deletion leads to neonatal lethality [52]. Here we reveal TET2 as a necessary factor for early key neural gene activation in hESC neural differentiation (Figures 4 and 7). These findings highlight a convergent role of TET proteins in neurodevelopment across species, and suggest that specific TET family members may be responsible for different developmental processes in different species [53].

TET family possesses only three members but catalyze DNA hydroxylation in the whole genome in a tissue-specific manner, suggesting specific mechanisms of their target specificities. It has been reported that the $\mathrm{N}$-terminal CXXC domain of Xenopus Tet 3 is required for DNA binding specificity with a slight preference for $\mathrm{CpG}$ content [38]. We also consistently reveal that human TET2, which lacks the CXXC domain, occupies at a C-rich element (Figure 5), while the binding sequences of both TET3 and TET 2 are not specific enough for TET $2 / 3$ targeting to specific neural gene loci. In agreement with previous hypothesis, TET DNA binding and enzymatic activity may be coordinated with its associated cofactors to modulate gene transcription [38]. This hypothesis is supported by the reports that neuronal Tet3 is recruited by REST for induction of target gene expression [54] and that TET enzymatic activation depends on interaction with CRL4 complex in primordial follicles [55]. Here we show that AF9 physically interacts with TET2 in vivo and in vitro (Figure 3) and that they collaborate to regulate $5 \mathrm{hmC}$ generation and activate neurodevelopmental genes (SOX5, ZNF521, MASH1, NEUROG1, and $M A P 2$ ) (Figures 6 and 7). Both AF9 and TET2 are MLL fusion partners that regulate $H O X$ gene transcription in hematological malignancies [56-58]. ChIP-seq and ChIP analysis revealed that AF9 and TET2 co-occupied the common neural-specific gene loci and that TET2 enrichment at these sites depends on AF9 recruitment (Figure 5). Functionally, the neural-promoting effect of AF9 is dependent on TET2 ability to promote $5 \mathrm{mC}$-to- $5 \mathrm{hmC}$ conversions at neurodevelopmental gene loci (Figures 4 and 7). This result is consistent with the role of TET2 in maintaining $H O X A$ activity through $5 \mathrm{mC}$ hydroxylation at lineage-specific loci [59].

DNA methylation dynamics are associated with brain development, maturation and synaptogenesis $[35,60]$. Consistently, a global epigenomic transition from $5 \mathrm{mC}$ enrichment in hESCs to $5 \mathrm{hmC}$ enrichment in neural cells was observed, correlating with neurogenesis and synapse formation (Figure 6; Supplementary Figure S6). 5hmC marks brain cell genomes in the fetal and adult brain [30, 31, 35, 37] and TET3-mediated $5 \mathrm{hmC}$ generation is an important epigenetic mechanism for master neural gene activation in Xenopus [38]. Importantly, CG demethylation at $5 \mathrm{hmC}$-poised loci in the genomes of mouse brain cells depends on Tet2 activity, and both $5 \mathrm{hmC}$ and TET2 have been observed in the human brain cortex $[26,35,37]$. These observations support our findings

Figure 7 AF9 and TET2 cooperate to regulate the conversion of $5 \mathrm{mC}$ to $5 \mathrm{hmC}$ at neural gene loci. (a) $5 \mathrm{hmC}$-positive cells were co-lobeled with TUJ1 in AF9-induced neurons. Control (Ctrl) or AF9-overexpressing (AF9-IRES-RFP) cells were differentiated for 12 days according to the protocol shown in Figure $2 \mathrm{~A}$ and co-stained with anti-TUJ1 and anti-5hmC antibodies. Bar, $100 \mu \mathrm{m}$. (b) Glu-qPCR analysis of $5 \mathrm{hmC}$ levels in day 8 or day 12 differentiated cells as indicated. Ctrl/D8, day 8 cells expressing control vector; AF9 OV/D8, day 8 cells expressing AF9-IRES-RFP; Ctrl KD/D12, day 12 cells expressing control shRNA; AF9 KD1/D12 or AF9 KD2/D12, day 12 cells expressing AF9 shRNA1 or shRNA2; Ctrl KD'/D12, day 12 cells expressing control shRNA; TET2 KD1/D12 or TET2 KD2/D12, day 12 cells expressing TET2 shRNA1 or shRNA2; AF9OV+Ctrl KD'/D8, day 8 cells expressing control shRNA and AF9-IRES-RFP; AF9OV+TET2 KD1/D8 or AF9OV+TET2 KD2/D8, day 8 cells expressing TET2 shRNA1 or shRNA2 and AF9-IRES-RFP. (c) DNA immunoprecipitation was performed using an anti-5mC antibody. DIP-qPCR analysis was conducted to determine the $5 \mathrm{mC}$ levels relative to input DNA in the same group of cells in (b). (d) A model for the role of the AF9-TET2 complex in the modulation of human embryonic stem cell (hESC) neural differentiation. In hESCs, some neural gene loci are methylated and inactive. During neural differentiation, upregulated AF9 are enriched in the neurodevelopmental gene loci by recognizing the AAC-containing elements. Then, AF9 recruits TET2 to nearby C-rich DNA sequences through the TET2 catalytic domain, and the AF9-TET2 complex cooperates to direct the $5 \mathrm{mC}$ conversion to $5 \mathrm{hmC}$ or to unmethylated cytosine (c). The AF9-TET2 complex-mediated $5 \mathrm{mC}$-to-5hmC conversions results in the activation of multiple neural genes and hESC differentiation into neurons. 
that TET2 has positive roles in hESC neural commitment through directing $5 \mathrm{mC}$ hydroxylation at neuralspecific gene loci (Figures 4 and 7). The C-terminal catalytic domain of human TET2 is responsible for DNA hydroxylation, DNA binding [61], and interaction with AF9 (Figure 3). Our study reveals a novel aspect of TET2 activity in human neural development, which relies on complex formation with AF9 that is enriched in sequence-specific motifs in neural-specific gene loci and provides recognition and recruitment signals for TET2. The specific expression of AF9 and TET2 during hESC neural differentiation is crucial for the establishment of an appropriate DNA hydroxylation profile and allow progressive neural commitment. Interestingly, the catalytic domain of TET2, which is important for overall structure formation of TET2 and TET2-DNA interactions [61], is important for its association with AF9 (Supplementary Figure S3C). It is possible that HXD motif mutation, which abolished enzymatic activity [61], may lead to the protein conformation change of C-terminal domain of TET2.

In addition, the striking effect of AF9 in promoting neuronal differentiation (Figure 2) was similar to the single-step induction of neurons observed upon forced expression of master neural regulators, such as MASH1 and NGN2 [62, 63]. The main difference is that the AF9TET2 complex sequentially activates the expression of endogenous NP genes, proneural genes and neuronal genes through epigenetic modulation of the $5 \mathrm{mC} / 5 \mathrm{hmC}$ status. Interference of DNA methylation disrupted the neural gene activation program (Supplementary Figure S1A and S6), indicating the importance of the AF9 TET2 complex for maintaining the well-balanced DNA modification profiles. In agreement with this notion, $A F 9$ mutations and disturbed DNA methylation or hydroxymethylation have been implicated in human neurodevelopmental disorders [18, 34, 64]. Our study provides an explanation why these genetic and epigenetic abnormalities may result in abnormal neural gene transcription and neurodevelopment.

In summary, our findings reveal a molecular model for the role of AF9-TET2 complex in neurodevelopment. This model involves the targeted recruitment of TET2 to $5 \mathrm{mC}$-containing genomic loci by AF9, followed by TET2-mediated hydroxylation that converts $5 \mathrm{mC}$ to $5 \mathrm{hmC}$ at neural-specific gene loci (Figure 7d). AF9 occupies the neurodevelopmental gene loci via recognizing motifs containing AAC nucleotides and, with its C-terminal region (Figure 3), recruits TET2 to the adjacent $\mathrm{C}$-rich regions at the loci. The recruited TET2 catalyzes the conversion of CD-bound $5 \mathrm{mC}$ to $5 \mathrm{hmC}$ during hESC neural differentiation and subsequently activates target gene expression to ensure normal and precisely controlled human neural development.

\section{Materials and Methods}

\section{Cell culture and treatment}

H9 hESC maintenance and neural differentiation were performed as previously described [5]. In brief, normally cultured hESCs in standard medium with bFgf were transferred to bFgf-free medium as aggregates (EBs) for 4 days. The EBs were then cultured in NP medium for another 2 days. Subsequently, the EBs were attached to the dish (called attached EB) and cultured in NP medium for 10 days. Then, the attached EBs were dissociated into patches and cultured as suspended neural spheres for 4 days. Finally, the neural spheres (NS) were trypsinized to single cells and replated onto culture dishes. HEK293T cells were cultured in DMEM medium supplemented with $10 \%$ FBS.

\section{Immunoprecipitation and co-immunoprecipitation}

Immunoprecipitation and co-immunoprecipitation assays were performed according to our previously described protocol [65]. The following antibodies were used: anti-AF9 (NB100-1566, Novus Biologicals, Littleton, CO, USA), antiMyc (Santa Cruz Biotechnology, Santa Cruz, CA, USA), and anti-Flag (Sigma, St Louis, MO, USA).

\section{Mass spectrometry}

The immunoprecipitation assay was performed as described above using day 12 cells differentiated from hESCs and anti-AF9 antibodies (NB100-1566, Novus Biologicals). The AF9-associated protein bands were subjected to in-gel digestion and mass spectrometric characterization [65] (Thermo Fisher Scientific, Waltham, MA, USA). The identified proteins are listed in Supplementary Table S1.

\section{Gene overexpression and knockdown in hESCs}

Lentivirus-mediated gene overexpression and knockdown in human H9 cells were performed as previously described [66], and the lentiviral infection method used was described in a published protocol [67]. Lentiviral infected clones were enriched by manual selection or sorted by FACS. The control and shRNA sequences are listed in Supplementary Table S2.

\section{$R N A$ extraction and $q P C R$ analysis}

Total RNA was extracted from cultured cells using TRIzol reagent (Pufei Bio, Shanghai, China). Reverse transcription and qPCR were performed as previously described [68]. All qPCR results were repeated for at least three times and one representative result was shown. The primers are listed in Supplementary Table S3.

\section{Half in vitro interaction assay}

His-AF9 was purified from BL21 (DE3) cells, cross-linked with Ni-agarose, and then incubated for $4 \mathrm{~h}$ at $4{ }^{\circ} \mathrm{C}$ with lysate from HEK293T cells expressing TET2-myc. After incubation and wash, the Ni-agarose was boiled at $100^{\circ} \mathrm{C}$ for $10 \mathrm{~min}$, and the eluted samples were loaded for SDS-PAGE analysis. 
ChIP-seq

ChIP was performed as previously described [68] using anti-AF9 (NB100-1566, Novus Biologicals) and anti-TET2 (R1086-1b, Abiocode, Agoura Hills, CA, USA) antibodies with day 12 cells (GEO: GSE68332). The high-throughput sequencing was performed by the Computational Biology Omics Core (CAS-MPG Partner Institute for Computational Biology, China).

\section{MeDIP/hMeDIP-seq and DIP-qPCR}

Genomic DNA was purified from hESCs and day 12 differentiated cells and sonicated. MeDIP and hMeDIP were performed as previously described [47] using anti-5mC (39649, Active Motif, Carlsbad, CA, USA) or anti-5hmC antibodies (MABE317, Millipore, Darmstadt, Germany) (GEO: GSE68396). The immunoprecipitated DNA was sequenced by the Computational Biology Omics Core (CAS-MPG Partner Institute for Computational Biology, China). To detect the $5 \mathrm{mC}$ levels, the immunoprecipitated DNA using an anti-5mC antibody and input DNA were subjected to qPCR analysis. Then input $\%$ was calculated and assigned as the relative $5 \mathrm{mC}$ level.

\section{Glu-qPCR}

The quantitative level of $5 \mathrm{hmC}$ was determined by Glu-qPCR [43] using the Quest 5-hmC Detection Kit (Zymo Research, Orange, CA, USA) according to the manufacturer's instructions. The primers used for ChIP verification and Glu-qPCR are listed in Supplementary Table S4. The primers used for ChIP verification, DIP-qPCR and Glu-qPCR are listed in Supplementary Table S4.

\section{Conflict of Interest}

The authors declare no conflict of interest.

\section{Acknowledgements}

This work was supported in part by the 'Strategic Priority Research Program' of the Chinese Academy of Sciences, Grant No. XDA01010201, National Key Basic Research and Development Program of China (2014CB964804, 2015CB964500), National Natural Science Foundation of China (91219303, 31430058, 31401261), and Chinese Postdoctoral Science Foundation (2013M541560).

\section{References}

1 Nadarajah B, Parnavelas JG. Modes of neuronal migration in the developing cerebral cortex. Nat Rev Neurosci 2002; 3 : 423-432.

2 Letinic K, Zoncu R, Rakic P. Origin of GABAergic neurons in the human neocortex. Nature 2002; 417: 645-649.

3 Sidman RL, Rakic P. Neuronal migration, with special reference to developing human brain: a review. Brain Res 1973; 62: 1-35.
4 Zhang SC, Wernig M, Duncan ID, Brustle O, Thomson JA. In vitro differentiation of transplantable neural precursors from human embryonic stem cells. Nat Biotechnol 2001; 19: 1129-1133.

5 Pankratz MT, Li XJ, Lavaute TM, Lyons EA, Chen X, Zhang SC. Directed neural differentiation of human embryonic stem cells via an obligated primitive anterior stage. Stem Cells 2007; 25: 1511-1520.

6 Vilchez D, Boyer L, Lutz M et al. FOXO4 is necessary for neural differentiation of human embryonic stem cells. Aging cell 2013; 12: 518-522.

7 Cimadamore F, Amador-Arjona A, Chen C, Huang CT, Terskikh AV. SOX2-LIN28/let-7 pathway regulates proliferation and neurogenesis in neural precursors. Proc Natl Acad Sci USA2013; 110: E3017-E3026.

8 Guillemot F. Spatial and temporal specification of neural fates by transcription factor codes. Development 2007; 134: 3771-3780.

9 Lai T, Jabaudon D, Molyneaux BJ et al. SOX5 controls the sequential generation of distinct corticofugal neuron subtypes. Neuron 2008; 57: 232-247.

10 Martinez-Morales PL, Quiroga AC, Barbas JA, Morales AV. SOX 5 controls cell cycle progression in neural progenitors by interfering with the WNT-beta-catenin pathway. EMBO Rep 2010; 11: 466-472.

11 Kamiya D, Banno S, Sasai N et al. Intrinsic transition of embryonic stem-cell differentiation into neural progenitors. Nature 2011; 470: 503-509.

12 Zhang X, Huang CT, Chen J et al. Pax6 is a human neuroectoderm cell fate determinant. Cell Stem Cell 2010; 7: 90-100.

13 Akbarian S, Huang HS. Epigenetic regulation in human brain-focus on histone lysine methylation. Biol Psychiatr 2009; 65: 198-203.

14 Buttner N, Johnsen SA, Kugler S, Vogel T. Af9/Mllt3 interferes with Tbr1 expression through epigenetic modification of histone H3K79 during development of the cerebral cortex. Proc Natl Acad Sci USA 2010; 107: 7042-7047.

15 Collins EC, Appert A, Ariza-McNaughton L, Pannell R, Yamada Y, Rabbitts TH. Mouse Af9 is a controller of embryo patterning, like Mll, whose human homologue fuses with Af9 after chromosomal translocation in leukemia. Mol Cell Biol 2002; 22: 7313-7324.

16 Vogel T, Gruss P. Expression of leukaemia associated transcription factor Af9/Mllt3 in the cerebral cortex of the mouse. Gene Expr Patterns 2009; 9: 83-93.

17 Striano P, Elia M, Castiglia L, Galesi O, Pelligra S, Striano S. A t (4;9)(q34;p22) translocation associated with partial epilepsy, mental retardation, and dysmorphism. Epilepsia 2005; 46: 1322-1324.

18 Pramparo T, Grosso S, Messa J et al. Loss-of-function mutation of the AF9/MLLT3 gene in a girl with neuromotor development delay, cerebellar ataxia, and epilepsy. Hum Genet 2005; 118: 76-81.

19 Srinivasan RS, de Erkenez AC, Hemenway CS. The mixed lineage leukemia fusion partner AF9 binds specific isoforms of the BCL-6 corepressor. Oncogene 2003; 22: 3395-3406. 
20 Erfurth F, Hemenway CS, de Erkenez AC, Domer PH. MLL fusion partners AF4 and AF9 interact at subnuclear foci. Leukemia 2004; 18: 92-102.

21 Hemenway CS, de Erkenez AC, Gould GC. The polycomb protein MPc3 interacts with AF9, an MLL fusion partner in $\mathrm{t}(9 ; 11)(\mathrm{p} 22 ; \mathrm{q} 23)$ acute leukemias. Oncogene 2001; 20: 3798-3805.

22 Zhang W, Xia X, Reisenauer MR, Hemenway CS, Kone BC. Dotla-AF9 complex mediates histone H3 Lys-79 hypermethylation and repression of ENaCalpha in an aldosterone-sensitive manner. J Biol Chem 2006; 281: 18059-18068.

23 Bitoun E, Oliver PL, Davies KE. The mixed-lineage leukemia fusion partner AF4 stimulates RNA polymerase II transcriptional elongation and mediates coordinated chromatin remodeling. Hum Mol Genet 2007; 16: $92-106$.

24 Cierpicki T, Risner LE, Grembecka J et al. Structure of the MLL CXXC domain-DNA complex and its functional role in MLL-AF9 leukemia. Nat Struct Mol Biol 2010; 17: 62-68.

$25 \mathrm{Wu} \mathrm{H}$, Coskun V, Tao J et al. Dnmt3a-dependent nonpromoter DNA methylation facilitates transcription of neurogenic genes. Science 2010; 329: $444-448$.

26 Siegmund KD, Connor CM, Campan $\mathrm{M}$ et al. DNA methylation in the human cerebral cortex is dynamically regulated throughout the life span and involves differentiated neurons. PLoS ONE 2007; 2: e895.

27 Ladd-Acosta C, Pevsner J, Sabunciyan S et al. DNA methylation signatures within the human brain. Am J Hum Genet 2007; 81: 1304-1315.

28 Tahiliani M, Koh KP, Shen Y et al. Conversion of 5-methylcytosine to 5-hydroxymethylcytosine in mammalian DNA by MLL partner TET1. Science 2009; 324: 930-935.

29 Wu H, Zhang Y. Mechanisms and functions of Tet proteinmediated 5-methylcytosine oxidation. Gene Dev 2011; 25: 2436-2452.

30 Zhang RR, Cui QY, Murai K et al. Tet1 regulates adult hippocampal neurogenesis and cognition. Cell Stem Cell 2013; 13: 237-245.

31 Guo JU, Su Y, Zhong C, Ming GL, Song H. Hydroxylation of 5-methylcytosine by TET1 promotes active DNA demethylation in the adult brain. Cell 2011; 145: 423-434.

32 Kriaucionis S, Heintz N. The nuclear DNA base 5-hydroxymethylcytosine is present in Purkinje neurons and the brain. Science 2009; 324: 929-930.

33 Szulwach KE, Li X, Li Y et al. 5-hmC-mediated epigenetic dynamics during postnatal neurodevelopment and aging. Nat Neurosci 2011; 14: 1607-1616.

34 Wang $\mathrm{T}$, Pan Q, Lin L et al. Genome-wide DNA hydroxymethylation changes are associated with neurodevelopmental genes in the developing human cerebellum. Hum Mol Genet 2012; 21: 5500-5510.

35 Lister R, Mukamel EA, Nery JR et al. Global epigenomic reconfiguration during mammalian brain development. Science 2013; 341: 1237905.
36 Ma DK, Jang MH, Guo JU et al. Neuronal activity-induced Gadd45b promotes epigenetic DNA demethylation and adult neurogenesis. Science 2009; 323: 1074-1077.

37 Orr BA, Haffner MC, Nelson WG, Yegnasubramanian S, Eberhart CG. Decreased 5-hydroxymethylcytosine is associated with neural progenitor phenotype in normal brain and shorter survival in malignant glioma. PLOS ONE 2012; 7: e41036.

$38 \mathrm{Xu} \mathrm{Y,} \mathrm{Xu} \mathrm{C,} \mathrm{Kato} \mathrm{A} \mathrm{et} \mathrm{al.} \mathrm{Tet3} \mathrm{CXXC} \mathrm{domain} \mathrm{and}$ dioxygenase activity cooperatively regulate key genes for Xenopus eye and neural development. Cell 2012; 151: 1200-1213.

39 Qiao Y, Wang R, Yang X, Tang K, Jing N. Dual roles of histone $\mathrm{H} 3$ lysine 9 acetylation in human embryonic stem cell pluripotency and neural differentiation. $J$ Biol Chem 2015; 290: 2508-2520.

40 Lin JJ, Hemenway CS. Hsp90 directly modulates the spatial distribution of AF9/MLLT3 and affects target gene expression. J Biol Chem 2010; 285: 11966-11973.

41 Ko M, Huang Y, Jankowska AM et al. Impaired hydroxylation of 5-methylcytosine in myeloid cancers with mutant TET2. Nature 2010; 468: 839-843.

42 Mercer TR, Dinger ME, Sunkin SM, Mehler MF, Mattick JS. Specific expression of long noncoding RNAs in the mouse brain. Proc Natl Acad Sci USA 2008; 105: 716-721.

43 Hackett JA, Sengupta R, Zylicz JJ et al. Germline DNA demethylation dynamics and imprint erasure through 5-hydroxymethylcytosine. Science 2013; 339: 448-452.

44 Vincent JJ, Huang Y, Chen PY et al. Stage-specific roles for tet1 and tet2 in DNA demethylation in primordial germ cells. Cell Stem Cell 2013; 12: 470-478.

45 Shen L, Wu H, Diep D et al. Genome-wide analysis reveals TET- and TDG-dependent 5-methylcytosine oxidation dynamics. Cell 2013; 153: 692-706.

46 Nguyen AT, Taranova O, He J, Zhang Y. DOT1L, the H3K79 methyltransferase, is required for MLLAF9-mediated leukemogenesis. Blood 2011; 117: 6912-6922.

$47 \mathrm{Xu} \mathrm{Y,} \mathrm{Wu} \mathrm{F,} \mathrm{Tan} \mathrm{L} \mathrm{et} \mathrm{al.} \mathrm{Genome-wide} \mathrm{regulation} \mathrm{of}$ $5 \mathrm{hmC}, 5 \mathrm{mC}$, and gene expression by Tet1 hydroxylase in mouse embryonic stem cells. Mol Cell 2011; 42: 451-464.

48 Koh KP, Yabuuchi A, Rao S et al. Tet1 and Tet2 regulate 5-hydroxymethylcytosine production and cell lineage specification in mouse embryonic stem cells. Cell Stem Cell 2011; 8: 200-213.

49 Li T, Yang D, Li J, Tang Y, Yang J, Le W. Critical role of Tet3 in neural progenitor cell maintenance and terminal differentiation. Mol Neurobiol 2015; 51: 142-154.

50 Dawlaty MM, Ganz K, Powell BE et al. Tet1 is dispensable for maintaining pluripotency and its loss is compatible with embryonic and postnatal development. Cell Stem Cell 2011; 9: 166-175.

51 Li Z, Cai X, Cai CL et al. Deletion of Tet2 in mice leads to dysregulated hematopoietic stem cells and subsequent development of myeloid malignancies. Blood 2011; 118: 4509-4518. 
$52 \mathrm{Gu}$ TP, Guo F, Yang $\mathrm{H}$ et al. The role of Tet3 DNA dioxygenase in epigenetic reprogramming by oocytes. Nature 2011; 477: 606-610.

53 Langemeijer SM, Aslanyan MG, Jansen JH. TET proteins in malignant hematopoiesis. Cell Cycle 2009; 8: 4044- 4048.

54 Perera A, Eisen D, Wagner M et al. TET3 Is Recruited by REST for Context-Specific Hydroxymethylation and Induction of Gene Expression. Cell Rep 2015; 11: 283-294.

55 Yu C, Zhang YL, Pan WW et al. CRL4 complex regulates mammalian oocyte survival and reprogramming by activation of TET proteins. Science 2013; 342: 1518-1521.

56 Kosmider O, Gelsi-Boyer V, Ciudad M et al. TET2 gene mutation is a frequent and adverse event in chronic myelomonocytic leukemia. Haematologica 2009; 94: 1676-1681.

57 Cheung N, So CW. Transcriptional and epigenetic networks in haematological malignancy. FEBS Lett 2011; 585: 2100-2111.

58 Solary E, Bernard OA, Tefferi A, Fuks F, Vainchenker W. The Ten-Eleven Translocation-2 (TET2) gene in hematopoiesis and hematopoietic diseases. Leukemia 2014; 28 : 485-496.

59 Bocker MT, Tuorto F, Raddatz G et al. Hydroxylation of 5-methylcytosine by TET2 maintains the active state of the mammalian HOXA cluster. Nat Commun 2012; 3: 818.

60 Numata S, Ye T, Hyde TM et al. DNA methylation signatures in development and aging of the human prefrontal cortex. Am J Hum Genet 2012; 90: 260-272.

$61 \mathrm{Hu} \mathrm{L}, \mathrm{Li} \mathrm{Z}$, Cheng J et al. Crystal structure of TET2-DNA complex: insight into TET-mediated $5 \mathrm{mC}$ oxidation. Cell 2013; 155: 1545-1555.

62 Pang ZP, Yang N, Vierbuchen T et al. Induction of human neuronal cells by defined transcription factors. Nature 2011; 476: $220-223$.
63 Zhang Y, Pak C, Han Y et al. Rapid single-step induction of functional neurons from human pluripotent stem cells. Neuron 2013; 78: 785-798.

64 Amir RE, Van den Veyver IB, Wan M, Tran CQ, Francke U, Zoghbi HY. Rett syndrome is caused by mutations in X-linked MECP2, encoding methyl-CpGbinding protein 2. Nat Genet 1999; 23: 185-188.

65 Yuan ZL, Guan YJ, Chatterjee D, Chin YE. Stat3 dimerization regulated by reversible acetylation of a single lysine residue. Science 2005; 307: 269-273.

66 Qiao Y, Zhu Y, Sheng N et al. AP2gamma regulates neural and epidermal development downstream of the BMP pathway at early stages of ectodermal patterning. Cell Res 2012; 22: 1546-1561.

67 Tulpule A, Daley GQ. Efficient gene knockdowns in human embryonic stem cells using lentiviral-based RNAi. Methods Mol Biol 2009; 482: 35-42.

68 Jin Z, Liu L, Bian W et al. Different transcription factors regulate nestin gene expression during P19 cell neural differentiation and central nervous system development. J Biol Chem 2009; 284: 8160-8173.

(Supplementary Information is linked to the online version of the paper on the Cell Discovery website.)

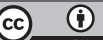

This work is licensed under a Creative Commons Attribution 4.0 International License. The images or other third party material in this article are included in the article's Creative Commons license, unless indicated otherwise in the credit line; if the material is not included under the Creative Commons license, users will need to obtain permission from the license holder to reproduce the material. To view a copy of this license, visit http://creativecommons.org/licenses/by/4.0/ 